\title{
Testing Hypotheses About Direction of Causation Using Cross-Sectional Family Data
}

\author{
A. C. Heath, ${ }^{1}$ R. C. Kessler, ${ }^{2}$ M. C. Neale, ${ }^{3}$ J. K. Hewitt, ${ }^{3}$ L. J. Eaves, ${ }^{3,4}$ and \\ K. S. Kendler ${ }^{3,4}$
}

Received 2 Apr. 1992-Final 28 May 1992

We review the conditions under which cross-sectional family data (e.g., data on twin
pairs or adoptees and their adoptive and biological relatives) are informative about di-
rection of causation. When two correlated traits have rather different modes of inheritance
(e.g., family resemblance is determined largely by family background for one trait and
by genetic factors for the other trait), cross-sectional family data will allow tests of strong
unidirectional causal hypotheses (A and B are correlated "because of the causal influence
of A on B" versus "because of the causal influence of B on A") and, under some
conditions, also of the hypothesis of reciprocal causation. Possible sources of errors of
inference are considered. Power analyses are reported which suggest that multiple indi-
cator variables will be needed to ensure adequate power of rejecting false models in the
presence of realistic levels of measurement error. These methods may prove useful in
cases where conventional methods to establish causality, by intervention, by prospective
study, or by measurement of instrumental variables, are infeasible economically, ethically
or practically.

KEY WORDS: Twins; reciprocal causation; genetics.

\section{INTRODUCTION}

It is widely acknowledged that the existence of a correlation between two variables, measured at a single point in time, has no necessary implications about causation (Fisher, 1958). There are many examples in the behavioral sciences where the direction of causation underlying an association between two variables is uncertain, and where reciprocal causation must be suspected. The existence of an association between measures of psychopathology (variable $A$ ) and perceived early childhood environment (variable $B$ ), for example, might arise because

\footnotetext{
${ }^{1}$ Departments of Psychiatry, Psychology, and Genetics, Washington University School of Medicine, 4940 Audubon Avenue, St. Louis, Missouri 63130.

2 Institute for Social Research, University of Michigan, Ann Arbor, Michigan 48109.

3 Department of Human Genetics, Medical College of Virginia, Richmond, Virginia 23298-0003.

4 Department of Psychiatry, Medical College of Virginia, Richmond, Virginia 23298-0003.
}

the early environment has a direct causal influence on risk of psychopathology $(B \rightarrow A)$ or because current psychopathology is biasing recall of early experiences $(A \rightarrow B)$, or alternatively, both these processes may be operating simultaneously (reciprocal causation: $A \rightleftarrows B$ ) (Martin and Heath, 1991; Neale et al., 1993). There may even be no direct causal link between the two variables, with each being influenced by other, unmeasured variables $(A \leftarrow C \rightarrow B)$ : inherited temperamental variables, for example, might lead both to a disruption of a child's early environment and to an increased risk of later psychopathology (cf. Bell, 1968). In this paper, we explore the conditions under which even cross-sectional data, on family members, can be used to test such causal hypotheses and review the limitations of this approach. We also explore the sample sizes needed for adequate statistical power to resolve alternative causal hypotheses, for a few instructive parameter sets, to determine how useful it is likely to be in practice. 


\section{Instrumental Variables}

To understand how family data can be used to test causal hypotheses, it is helpful to consider alternative research strategies, using observational (i.e., nonexperimental) data, that have been used. Instrumental variable methods (Fig. 1), widely used in econometrics (e.g., Johnston, 1972), have been advocated as one useful approach (Kessler, 1983). The path diagram (Wright, 1968) in Fig. 1 represents a reciprocal causal relationship between two variables, $A$ and $B$, which are also partly determined by "instruments"' $L A$, and $I B$, respectively, plus residual factors $U A$ and $U B$. The successful identification of instruments, i.e., variables which are related causally to one, and only one, of the two variables whose reciprocal causal influence is under study, is critical for this approach; and the mistaken assumption that a variable is an instrument when it in fact influences both the reciprocally interacting variables may lead to mistaken inferences about causality (Kessler, 1983). Also critical

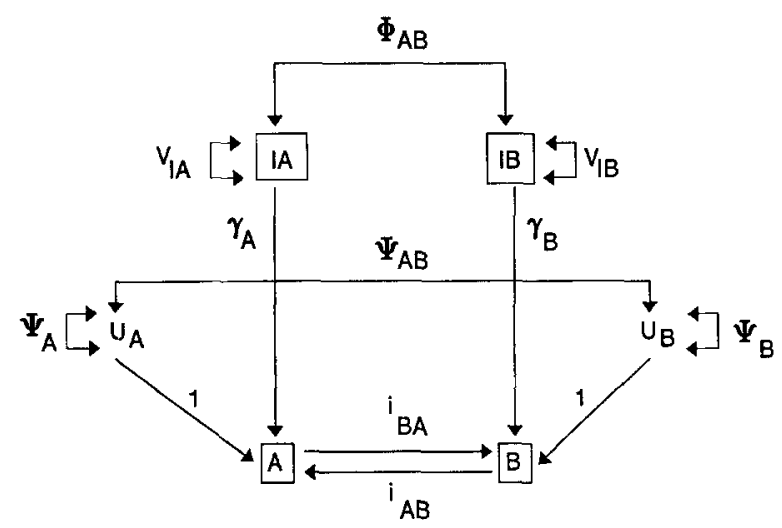

Fig. 1. Path diagram (Wright, 1968) representing an instrumental variable model for resolving reciprocal causal influences of two variables, $A$ and $B$, on each other. $A$ and $B$ are determined by exogenous instruments $I A$ and $I B$, plus residuals $U A$ and $U B$. Two-headed arrows between $L A$ and $I B$, and between $U A$ and $U B$, are used to denote the covariances of those variables. Two-headed arrows from a variable to itself are used to denote the variance of that variable. Single-headed arrows are used to denote the effects of exogenous variables on the endogenous variables $A$ and $B$, as well as of the endogenous variables on each other. Lowercase letters are used to represent model parameters corresponding to the covariances and variances of the exogenous variables, and path coefficients representing the direct effects on endogenous variables of exogenous and other exogenous variables, except in cases where these are fixed to particular numeric values. Path $i_{\mathrm{BA}}$ represents the direct causal influence of $A$ on $B$; path $i_{\mathrm{AB}}$ represents that of $B$ on $A$. is the assumption that residual effects on variable $A(U A)$ are uncorrelated with the instrument for variable $B(I B)$, and likewise that there is no correlation between $U B$ and $I A$.

Under the full model in Fig. 1, the expected covariance of variable $A$ with its instrument $I A$ is $s\left(\gamma_{\mathrm{A}} V_{\mathrm{IA}}+i_{\mathrm{BA}} \gamma_{\mathrm{B}} \phi_{\mathrm{AB}}\right)$, the covariance of $B$ with $I B$ is $s\left(\gamma_{\mathrm{B}} V_{\mathrm{IB}}+i_{\mathrm{BA}} \gamma_{\mathrm{A}} \phi_{\mathrm{AB}}\right)$, the covariance of $A$ with $I B$ is $s\left(\gamma_{\mathrm{A}} \phi_{\mathrm{AB}}+i_{\mathrm{AB}} \gamma_{\mathrm{B}} V_{\mathrm{IB}}\right)$, and the covariance of $B$ and $I A$ is $s\left(\gamma_{\mathrm{B}} \phi_{\mathrm{AB}}+i_{\mathrm{BA}} \gamma_{\mathrm{A}} V_{\mathrm{AA}}\right)$, where $s=\left(1-i_{\mathrm{AB}} i_{\mathrm{BA}}\right)^{-1}$. In the absence of any direct casual influence of $A$ on $B$ or of $B$ on $A\left(i_{\mathrm{AB}}=i_{\mathrm{BA}}=0\right)$, these covariances reduce to $\gamma_{\mathrm{A}} V_{\mathrm{IA}}, \gamma_{\mathrm{B}} V_{\mathrm{IB}}, \gamma_{\mathrm{A}} \phi_{\mathrm{AB}}$, and $\gamma_{\mathrm{B}} \phi_{\mathrm{AB}}$, respectively. In other words, in the absence of any direct influence of $A$ on $B$ or $B$ on $A$, the covariance of $A$ with $I B$, and of $B$ with $I A$, can be predicted from the variances and covariance of the instruments $L A$ and $I B$ and the covariance of $A$ with $I A$, or $B$ with $I B$, respectively. In the case of unidirectional causation, where $A$ is a cause of $B\left(A \rightarrow B\right.$, i.e., $i_{\mathrm{BA}} \neq 0, i_{\mathrm{AB}}$ $=0$ ), the covariance of $A$ with $I B$ does not change, but the covariance of $B$ with $L A$ changes to $\left(\gamma_{\mathrm{B}} \phi_{\mathrm{AB}}\right.$ $\left.+i_{\mathrm{BA}} \gamma_{\mathrm{A}} V_{\mathrm{IA}}\right)$; and conversely, under unidirectional causation with $B$ a cause of $A$, only the covariance of $A$ with $I B$ will change. Thus it is the cross-covariances between variable $A$ and the instrument for variable $B$, and between variable $B$ and the instrument for variable $A$, which provide critical information about the causal influence of $A$ on $B$ and $B$ on $A$.

The full model in Fig. 1 has 10 parameters and so will give a perfect fit to the $4 \times 4$ covariance matrix of variables $A, B, L A$, and $I B$. Thus there is a single degree of freedom available for testing the submodel with $\psi_{\mathrm{AB}}=0$, i.e., the hypothesis that there is no residual correlation between $A$ and $B$, after allowing for both the correlation between the instruments for $A$ and $B, \phi_{\mathrm{AB}}$, and the direct causal influence of $A$ on $B$ and $B$ on $A$, or for testing either unidirectional causal model and two degrees of freedom for testing the hypothesis of no direct causal influence of $A$ on $B$ or $B$ on $A$.

Despite the theoretical appeal of the instrumental variable model, examples in the behavioral sciences where it can be applied in practice have proved to be rare. It is seldom possible to be confident both that an instrument for $A$ has no direct causal influence on $B$ and that an instrument for $B$ has no direct causal influence on $A$. To take a concrete example, we may be interested in the reciprocal causal relationship between adolescent delinquent behavior and illicit substance use in a national survey. It might be possible to argue that 
a measure of neighborhood or community "drug availability" would be a potential instrument for the illicit substances use variable. It is extremely difficult to conceive of a suitable instrumental variable for delinquent behavior which could be assumed, with any degree of confidence, to have no direct effect on illicit substance use. Furthermore, it is easy to imagine that certain unmeasured neighborhood characteristics might lead both to increased drug availability and use and to increased risk of delinquent behavior, violating the assumption of no correlation between the residual for variable $B$ and the instrument for variable $A$.

It is sometimes possible to identify an instrument for only one variable, which at least makes possible a test of a unidirectional causal hypothesis. For example, if we are interested in the relationship between adolescent smoking and illicit substance use (e.g., Yamaguchi and Kandel, 1984) or between smoking and depression (e.g., Breslau, 1991; Kendler et al., 1992), the assumption that regional differences in the sales tax on cigarettes will be an appropriate instrument for smoking, and uncorrelated with the residual for depression (or illicit substance use), may sometimes be justifiable, although not if both variables are influenced by local economic conditions. In these circumstances, the hypothesis of no direct causal influence of smoking on illicit substance use (or depression) predicts no covariance between sales tax and illicit substance use (or depression). However, in most examples in the behavioral sciences we must allow for the possibility of reciprocal causation. Another problem confronts the practical application of the instrumental variable model, even if instruments can be successfully identified: variables $A$ and $B$ and their instruments are unlikely to be measured without error (as is implicit in Fig. 1). However, this problem can often be overcome, by using multiple indicator variables (Bollen, 1989) to assess the underlying latent constructs of the model.

\section{Panel Data}

Many investigators have explored the use of longitudinal data, most commonly two-wave panel data, to address issues of causality (e.g., Campbell, 1963; Duncan, 1969) and have explored the critical underlying assumptions, violation of which may lead to errors of inference (e.g., Duncan, 1969; Kenny, 1975; Rogosa, 1980; Locasio, 1982). In early papers the relative magnitude of the cross-temporal cross-trait ("cross-lagged") correlations, i.e., the correlations of $A$ measured at wave $1\left(A_{t \prime}\right)$ with $B$ measured at wave $2\left(B_{t 2}\right)$, and of $B$ measured at wave $1\left(B_{i l}\right)$ with $A$ measured at wave $2\left(A_{t 2}\right)$, were used to infer the relative strengths of the causal influence of $A$ on $B$ versus $B$ on $A$ : a higher absolute cross-correlation between $A_{t 1}$ and $B_{t 2}$ than between $B_{t 1}$ and $A_{t 2}$, for example, was taken to imply a predominant causal influence of $A$ on $B$. This approach can be seriously misleading (see, e.g., the critique by Rogosa, 1980), even when it is recast as a problem of resolving alternative structural equation hypotheses. Errors of inference may arise because of such issues as (i) differences in measurement error for the two traits, or the existence of correlated errors of measurement for the two traits; (ii) inconsistency between the causal lags (i.e., the intervals between a change in $A$ and the change in $B$ which this produces, and vice versa) and the time lag between waves of measurement; and (iii) partial or complete determination of the correlation between the two traits by a third unmeasured "mediating" variable, $C$. Such factors can lead to a higher crosscorrelation between $A_{t 1}$ and $B_{t 2}$ even when the predominant causal influence is $B \rightarrow A$.

If we consider the instantaneous reciprocal causal influences of $A$ on $B$ and vice versa, we can view the two-wave panel as being a particular instance of an instrumental variable model, where measured of $A$ and $B$ at wave 1 function as instruments for $A$ and $B$, respectively, at wave 2 (Kessler, 1983): i.e., we can substitute $A_{t l}$ for $L A$ and $B_{t}$ for $I B$, and $A_{t 2}$ for $A$ and $B_{t 2}$ for $B$, in Fig. 1. Thus a critical assumption is that there is no direct influence of $A$ at wave 1 on $B$ at wave 2 , and no direct infuence of $B$ at wave 1 on $A$ at wave 2 (Kessler, 1983). Even if this assumption is satisfied, the approach will break down if $A$ and $B$ are perfectly or very highly stable over time or if there are serial correlations of errors in $A$, and in $B$, which can lead to seriously biased estimates of the reciprocal paths (Kessler and Greenberg, 1981).

\section{Behavioral Genetic Approach}

A third possible approach to testing causal hypotheses in observational data, which is much less widely recognized, utilizes data on family members, for example, pairs of monozygotic and dizygotic twins (Health et al., 1989a; Neale et al., 1989b, 1993; Duffy and Martin, 1993; Neale and Cardon, 1992), or adoptees and their adoptive and biological 
relatives. This approach does not require the successful identification and measurement of instrumental variables and can be used with cross-sectional data. It can be used even when two associated variables are, except for any measurement error, completely stable over time and, because even crosssectional data are informative, avoids the problems of serially correlated errors in panel data. This approach may be viewed as a special case of the instrumental variables method, where we are using genetically informative designs to identify the effects of latent instruments, i.e., the genotypes and environments which determine traits $A$ and $B$.

To understand the circumstances in which crosssectional family data are informative about direction of causation, it is instructive to consider a simplified, and somewhat extreme, example (cf. Heath and Martin, 1991a, b). Figure 2 represents a path diagram for two variables having a reciprocal causal influence, $A$ and $B$, measured on pairs of relatives, with direct additive and nonadditive (dominance or epistatic) genetic influences on trait $A$, and shared environmental influences on trait $B$, as well as withinfamily environmental effects on each trait. In this simplified example, the only genetic influences on trait $B$ are those mediated through the causal influence of trait $A$ on trait $B$, and the only shared en- vironmental influences on trait $A$ are those mediated through the causal influence of trait $B$ on trait $A$. Trait $A$, for example, might denote a heritable personality variable (Eaves et al., 1989), and trait $B$ a measure of perceived childhood environment (e.g., Parker, 1983, 1990). Differences in inherited temperament may elicit differences in childhood environment (personality $\rightarrow$ environment), which may in turn influence personality development (childhood environment $\rightarrow$ personality). Latent variables $E, G$, and $D$ can be thought of as representing residual environmental and genetic influences on trait $A$, after allowing for the causal influence of $B$ on $A$; and likewise, latent variables $E^{\prime}$ and $C^{\prime}$ can be viewed as the residual environmental influences on $B$, after allowing for the causal influence of $A$ on $B$. These latent genetic and environmental variables are all assumed to be mutually uncorrelated.

We consider two submodels of Fig. 2: (a) $i_{\mathrm{AB}}$ $=0, i_{\mathrm{BA}} \neq 0$, implying $A$ is a cause of $B(A \rightarrow$ $B$ ); and (b) $i_{\mathrm{BA}}=0, \mathrm{i}_{\mathrm{AB}} \neq 0$, implying $B \rightarrow A$. We may derive expectations for the variances and covariances of relatives, using the tracing rules of path analysis (Wright, 1968), modified to allow for the fact that some of our variables are nonstandardized; or equivalently, we may apply simple matrix algebra for deriving expected covariance matrices

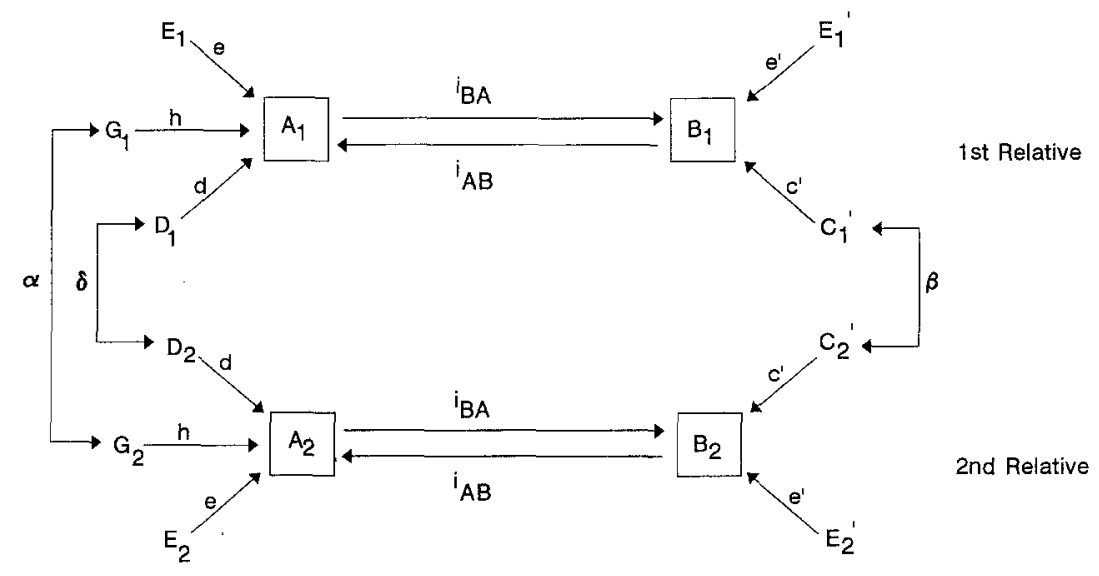

Fig. 2. Path diagram illustrating reciprocal interaction between two variables, $A$ and $B$, measured on pairs of relatives. Observed and latent variables are denoted by capital letters: endogenous variables $A$ and $B$ are the observed traits; exogenous variables $E$, $G$, and $D$ represent within-family environmental effects, additive genetic effects, and dominance genetic effects on $A$; and $E^{\prime}$ and $C^{\prime}$ denote within-family environmental effects and shared environmental effects on $B$. Subscripts are used to distinguish variables of first relative $\left(P A_{1}\right)$ from variables of second relative $\left(P A_{2}\right)$. Covariances $\alpha, \beta$, and $\delta$ denote the covariances of additive genetic effects, shared environmental effects, and dominance genetic effects, between relative pairs, which will vary as a function of genetic relatedness and rearing history. Omission of a two-headed arrow between exogenous variables indicates that covariance is fixed to zero ex hypothesi; omission of a two-headed arrow from an exogenous variable to itself is used to indicate that variable is standardized to unit variance. 
[e.g., using the LISREL model of Joreskog and Sorbom (1988); see also Bollen (1989)]. For the expectation for the cross-covariance of trait $A$ in relative $j\left(A_{j}\right)$, and trait $B$ in relative $k\left(B_{k}\right)$, in case (a: $A \rightarrow B$ ) we have $i_{\mathrm{BA}}\left(\alpha h^{2}+\delta d^{2}\right)$, and in case (b: $B \rightarrow A$ ) we have $i_{\mathrm{AB}} \beta c^{\prime 2}$. Thus $A \rightarrow B$, in this simplified example, predicts a positive cross-covariance between $A_{j}$ and $B_{k}$ in biological relatives, the magnitude of which will depend upon their degree of genetic relatedness, but a zero cross-covariance in adoptive relatives; whereas $B \rightarrow A$ predicts a positive cross-covariance which does not depend upon genetic relatedness for collateral relatives reared in the same family (e.g., MZ twin pairs, DZ twin pairs, biological or step siblings) and a zero correlation for relatives reared in separate families (e.g., separated twin pairs, biological mother and adoptedaway offspring).

Although this example represents an extreme case, the same considerations will apply when the relative magnitudes of direct genetic and shared environmental influences (or additive and dominance genetic influences) differ for the two traits. Thus if additive genetic effects are having a stronger direct influence than shared environmental effects on trait $A$, but shared environmental effects a stronger direct influence than genetic effects on trait $B$, then $A \rightarrow B$ predicts that the cross-covariance between $A_{j}$ and $B_{k}$ will be dominated by genetic effects, whereas $B \rightarrow A$ implies that it will be dominated by shared environmental effects. Similar to what we noted for instrumental variable or panel data, in family data we thus find that the cross-correlation between trait $A$ measured in one relative and trait $B$ measured in the second relative provides critical information about causality. However, we must note that in the case where the two traits have identical modes of inheritance (i.e., the proportions of the total variation accounted for by additive genetic, dominance genetic, shared environmental, and within-family environmental effects are identical for the two traits, so that correlations between relatives are the same for the two traits), then family data will be completely uninformative about direction of causation, as we discuss in greater detail later.

\section{METHOD}

\section{Basic Genetic Models and Assumptions}

Having considered at a somewhat intuitive level how cross-sectional data may be informative about direction of causation, we now consider this question more rigorously. Figure 3 summarizes, in the form of a composite path diagram (Wright, 1968), two multifactorial genetic models for the analysis of bivariate data (observed traits $Y_{\mathrm{A}}$ and $Y_{\mathrm{B}}$, which are measures of underlying latent variables or "phenotypes" $P_{\mathrm{A}}$ and $P_{\mathrm{B}}$ ): (i) a "general bivariate" model, which makes no assumptions about causality [fixing to zero the causal paths $i$ and $i^{\prime}$ (Eaves and Gale, 1974; Eaves and Eysenck, 1975; Heath et al., 1989a]; and (ii) a "direction-of-causation" model (fixing to zero the correlations between genetic and environmental effects on the two traits, $r_{\mathrm{E}}=r_{\mathrm{G}}=r_{\mathrm{C}}=r_{\mathrm{D}}=0$ ), which specifies that the only causes of the correlation between $P_{\mathrm{A}}$ and $B_{\mathrm{B}}$ are the causal influence of $P_{\mathrm{A}}$ on $P_{\mathrm{B}}$ (path $i^{\prime}$ ), or of $P_{\mathrm{B}}$ on $P_{\mathrm{A}}$ (path $i$ ), or the reciprocal causal influence of $P_{\mathrm{A}}$ and $P_{\mathrm{B}}$ on each other (Heath et al., 1989a; Neale et al., 1992). We use primes to distinguish genetic and environmental factors having a direct influence on trait $B\left(P_{B}\right)$ from those which have a direct effect on trait $A\left(P_{\mathrm{A}}\right)$; and decompose the total genetic effects on each trait into additive $\left(G\right.$ and $\left.G^{\prime}\right)$ and dominance $\left(D\right.$ or $\left.D^{\prime}\right)$ genetic effects, and the total environmental effects into family environmental effects, which are shared by collateral relatives reared in the same family $(C$ or $C^{\prime}$ ), and within-family environmental effects, which reflect differences in environmental experience even between individuals reared in the same family ( $E$ or $E^{\prime}$ ) (Jinks and Fulker, 1970; Eaves, 1977; Loehlin, 1978). Under the direction-of-causation model, there will be additional indirect effects of $E, G, C$, or $D$ on trait $B$, mediated through the causal influence of trait $A$ on trait $B$ (if $i^{\prime} \neq 0$ ), and of $E^{\prime}, G^{\prime}$, $C^{\prime}$, and $D^{\prime}$ on trait $A$, mediated through the causal influence of trait $B$ on trait $A$ (if $i \neq 0$ ). The general bivariate model, in contrast, allows for correlations between additive gene effects on $P_{\mathrm{A}}$ and $P_{\mathrm{B}}\left(r_{\mathrm{G}}\right)$, dominance gene effects on $P_{\mathrm{A}}$ and $P_{\mathrm{B}}\left(r_{\mathrm{D}}\right)$, withinfamily environmental effects on $P_{\mathrm{A}}$ and $P_{\mathrm{B}}\left(r_{\mathrm{E}}\right)$, and between-family or "shared" enviornmental effects on $P_{\mathrm{A}}$ and $P_{\mathrm{B}}\left(r_{\mathrm{C}}\right)$ and, thus, allows for the possibility that the two traits are correlated because they are both influenced by other, unmeasured traits, or by the same ("common," in the factor analytic sense) genetic or environmental risk factors.

We represent in Fig. 3 only the phenotypes and genetic and environmental deviations for a single individual: we do not attempt to represent pairs of relatives, since the resulting diagram is too complex to be useful. We use subscripts, $m$ and $n$, to 


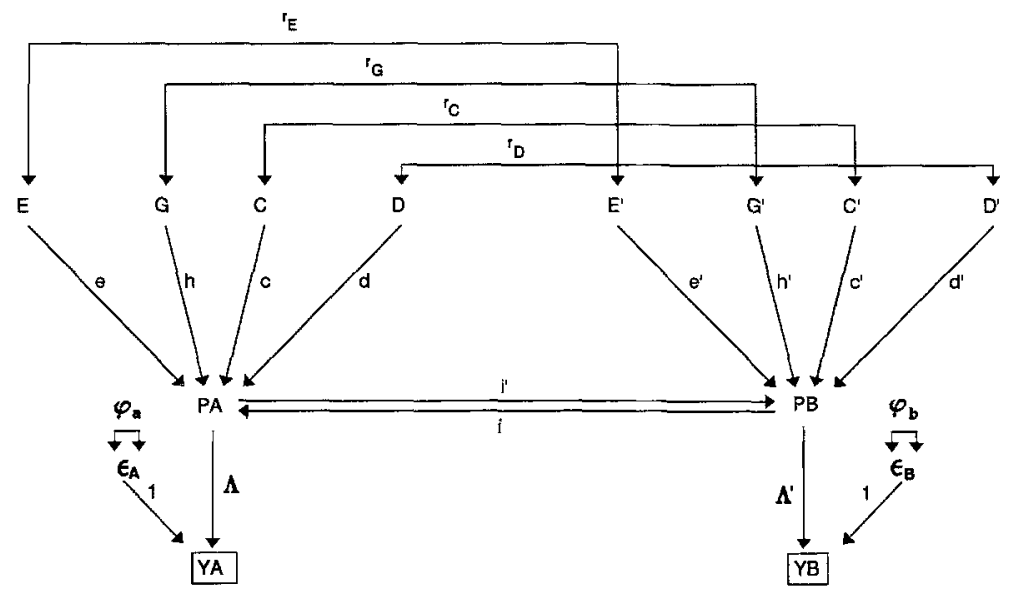

Fig. 3. Composite path diagram representing (i) general bivariate genetic model and (ii) "direction-of-causation" submodel. $Y A$ and $Y B$ denote observed variables (measured on a single individual), $P A$ and $P B$ denote the underlying latent traits which these are measuring, and $\epsilon_{\mathrm{A}}$ and $\epsilon_{\mathrm{B}}$ are the measurement errors for the observed variables. Covariances $r_{\mathrm{E}}, r_{\mathrm{G}}, r_{\mathrm{C}}$, and $r_{\mathrm{D}}$ denote covariances between within-family environmental effects on $P A$ and $P B$, between additive genetic effects, between shared environmental effects, and between dominance genetic effects. See Fig. 2 and text for definition of other model variables and parameters.

distinguish latent or observed variables of the $m$ th and $n$th relatives, e.g., with $m=1$ and $n=2$ for first and second twins from twin pairs of a given zygosity type. In terms of structural equations (e.g., Joreskog and Sorbom, 1988), from Fig. 3 we have

$$
\begin{aligned}
P_{\mathrm{A} m}= & h G_{m}+d D_{m}+c C_{m}+e E_{m}+i P_{\mathrm{B} m} \\
P_{\mathrm{B} m}= & h^{\prime} G_{m}^{\prime}+d^{\prime} D_{m}^{\prime}+c^{\prime} C_{m}^{\prime}+e^{\prime} E_{m}^{\prime} \\
& +i^{\prime} P_{\mathrm{A} m} \\
Y_{\mathrm{A} m}= & \lambda P_{\mathrm{A} m}+\epsilon_{\mathrm{A}} \\
Y_{\mathrm{B} m}= & \lambda^{\prime} P_{\mathrm{B} m}+\epsilon_{\mathrm{B}}
\end{aligned}
$$

with corresponding equations for the variables for the $n$th relative. Here latent genetic and environmental variables $(G, D, C, E$, etc.) are standardized to unit variance, and all variables scaled as deviations from zero. Genetic and environmental coefficients $h, d, c, e$, etc., and causal paths $i$ and $i^{\prime}$, are assumed to be the same in relatives of different types (e.g., MZ versus DZ twin pairs). The models in Fig. 3 include measurement error explicitly (error terms $\epsilon_{A}$ and $\epsilon_{B}$, with residual variances $\theta_{\epsilon A}$ and $\theta_{\epsilon A}$, respectively), since failure to allow for differences in error variance can easily lead to errors of inference about causality. In applications where we have only a single indicator variable corresponding to each phenotype, we will further fix $\lambda=\lambda^{\prime}=1$, but this constraint can be relaxed if we have multiple indicator variables, i.e., multiple measures for our constructs.

In order to derive expectations for the withintrait and cross-trait covariances of relative, we need values for the covariances of additive genetic, dominance genetic, shared environmental, and withinfamily environmental effects for each trait $\left(G_{m}\right.$, etc., and $G_{n}$, etc.), for different types of relationship. By definition, within-family environmental effects $\left(E\right.$ or $\left.E^{\prime}\right)$ are uncorrelated between relatives and, also, uncorrelated with familial effects $(C, G, D$, $C^{\prime}, G^{\prime}$, or $D^{\prime}$ ) for the same individual. Under the simplest model, the covariances between shared environmental effects $\left(C\right.$ or $\left.C^{\prime}\right)$ for the same trait (which we denote by $\beta$ ) will be unity for collateral relatives reared in the same family (e.g., monozygotic or dizygotic twin pairs or biological or adoptive sibling pairs) and zero otherwise, and correlations between relatives for shared environmental effects across traits will be $\beta r_{C}$ under the general bivariate model and zero under the direction-of-causation submodel. More elaborate environmental models may also be tested, provided that appropriate constellations of relatives are studied (e.g., Eaves et al., 1978; Fulker, 1982, 1988; Heath et al., 1985), models which could incorporate such complications as differences in similarity of environmental exposure of twins versus sibling pairs, parent-to-offspring environmental transmission, offspring-to- 
parent environmental transmission, nonrandom placement of adoptees, and reciprocal sibling environmental influences (Cavalli-Sforza and Feldman, 1981; Cloninger et al., 1979; Eaves, 1976; Eaves et al., 1978; Carey, 1986; Fulker, 1982; Heath, 1983; Heath et al., 1989a).

From genetic theory (Fisher, 1918; Falconer, 1960; Mather and Jinks, 1971; Bulmer, 1980), the correlation between the additive genetic deviations of relatives, within traits, which we denote $\alpha$, will be equal to the coefficient of relationship, under random mating, i.e., unity for monozygotic twin pairs, 0.5 for first-degree relatives, 0.25 for halfsibling pairs, etc., and zero for genetically unrelated individuals; and the correlation of dominance genetic effects $(\delta)$ will be unity for monozygotic twin pairs, 0.25 for dizygotic twin pairs, and zero for most other relationships; while the correlation between the additive genetic effects in one relative and dominance effects in the same relative or in another relative, will be zero. The corresponding correlation between additive genetic effects (or dominance effects) on trait $A$ in relative $m$ and additive genetic effects (or dominance effects) on trait $B$ in relative $n$ will be $\alpha r_{\mathrm{A}}$ (and $\delta r_{\mathrm{D}}$ ) under the general bivariate model but, again, zero under the direction-of-causation submodel. The assumption of random mating, i.e., that there is no tendency for like to marry like with respect to the variables under study, is easily relaxed (e.g. Fisher, 1918; Rice et al., 1978; Cloninger et al., 1979; Eaves et al., 1978; Heath and Eaves, 1985; Fulker, 1988; Cardon et al., 1991) but is retained here to simplify presentation. For the representation of the additive genetic variance $\left(h^{2}\right)$ and the dominance genetic variance $\left(d^{2}\right)$ in terms of allele frequencies and average allele effects at individual loci, see Mather and Jinks (1971). In principle, terms for epistatic interactions between genetic loci [additive $x$ additive, additive $\times$ dominance etc. (Mather and Jinks, 1971; Bulmer, 1980)] could be included in Eq. A. (1) and (2), but in practice resolution of the effects of genetic dominance and genetic epistasis for multifactorial traits, in human populations, requires extremely large sample sizes (Heath, 1983). In what follows, we make the additional simplifying assumptions of no genotype-environment correlation and no genotype $\times$ environment interaction, noting that these assumptions, too, can be relaxed if appropriate experimental designs are used (Eaves et al., 1977; Fulker, 1982; Heath et al., 1989a; Plomin et al., 1977).

\section{Hypothesis Testing}

The application of methods of structural equation modeling to testing genetic and environmental models, using twin and other family data, has been reviewed extensively elsewhere (Martin and Eaves, 1977; Eaves et al., 1978; Fulker, 1982; Heath et al., 1989a; Neale et al., 1989a). In the simplest case where data are available on collateral relative pairs (e.g., monozygotic and dizygotic twin pairs reared together and twin pairs reared apart), separate summary covariance or correlation matrices are computed for each group of relatives, and a series of nested models is fitted, in a multiple-group analysis, by maximum-likelihood (Joreskog and Sorbom, 1988), asymptotic weighted least-squares (Browne, 1984), or other fitting functions, yielding efficient estimates of model parameters. Expected covariance matrices will differ for different groups of relatives because of differences in the covariances of genetic and environmental factors (i.e., constants $\alpha, \beta$, and $\delta$ ), but genetic and environmental parameters $h, h^{\prime}, d, d^{\prime}$, and etc., are constrained to be equal across different relative groups, as well as between first and second members of same-sex relative pairs. A chi-square statistic is used to assess the overall goodness of fit of a model, and likelihood-ratio or chi-square difference tests are used to compare the fit of a general model with nested submodels, in order to identify the most parsimonious model consistent with the data (Joreskog, 1978; Eaves et al., 1978; Neale et al., 1989b). When more elaborate experimental designs are used, such as studying adoptees and their biological and adoptive siblings and parents (e.g., Fulker and DeFries, 1983), or twins reared together and their siblings and offspring (e.g., Eaves et al., 1992), the number of different family structures may be almost as large as the number of families, requiring that models be fitted directly to the observed raw data by maximum-likelihood (e.g., Lange et al., 1976; Eaves et al., 1978, 1989). In such analyses likelihood-ratio comparisons of the fit of a general model and submodels are made in the usual fashion.

We have described the full model in Fig. 3 as a "composite model," in order to recognize that the full model is indeterminate. In fact, it can be shown that the direction-of-causation model is a submodel of the general bivariate genetic model. To avoid confusion, we use subscripts $a$ and $b$ for genetic and environmental parameters under the general bivariate model (i.e., $h_{\mathrm{a}}$, etc., to replace $h$, 
etc., and $h_{\mathrm{b}}$, etc. to replace $h^{\prime}$, etc., in Fig. 3). Table I summarizes expected covariances of relatives under the two models. If the "true" model is the reciprocal causation model, our estimates of genetic and environmental variance components under the general bivariate model will be of the form $h_{\mathrm{a}}{ }^{2}=\left(h^{2}+i^{2} h^{\prime 2}\right)\left(1-i i^{\prime}\right)^{-2}, d_{\mathrm{a}}^{2}=\left(d^{2}+i^{2} d^{\prime 2}\right)$ $\left(1-i i^{\prime}\right)^{-2}, c_{\mathrm{a}}^{2}=\left(c^{2}+i^{2} c^{\prime 2}\right)\left(1-i i^{\prime}\right)^{-2}$, and $e_{\mathrm{a}}^{2}=\left(e^{2}+i^{2} e^{\prime 2}\right)\left(1-i i^{\prime}\right)^{-2}$ and $h_{\mathrm{b}}^{2}=\left(h^{\prime 2}+\right.$ $\left.i^{\prime 2} h^{2}\right)\left(1-i i^{\prime}\right)^{-2}, d_{\mathrm{b}}^{2}=\left(d^{\prime 2}+i^{2} d^{2}\right)\left(1-i i^{\prime}\right)^{-2}$, etc.; and estimates of correlations between gene effects or environmental effects will be

$$
\begin{aligned}
r_{\mathrm{G}}= & \left(i^{\prime} h^{2}+i h^{\prime 2}\right)\left(h^{2}+i^{2} h^{\prime 2}\right)^{-0.5} \\
& \left(h^{\prime 2}+i^{\prime 2} h^{2}\right)^{-0.5} \\
r_{\mathrm{D}}= & \left(i^{\prime} d^{2}+i d^{\prime 2}\right)\left(d^{2}+i^{2} d^{\prime 2}\right)^{-0.5} \\
& \left(d^{\prime 2}+i^{\prime 2} d^{2}\right)^{-0.5} \\
r_{\mathrm{C}}= & \left(i^{\prime} c^{2}+i c^{\prime 2}\right)\left(c^{2}+i^{2} c^{\prime 2}\right)^{-0.5} \\
& \left(c^{\prime 2}+i^{\prime 2} c^{2}\right)^{-0.5} \\
r_{\mathrm{E}}= & \left(i^{\prime} e^{2}+i e^{\prime 2}\right)\left(e^{2}+i^{2} e^{\prime 2}\right)^{-0.5} \\
& \left(e^{\prime 2}+i^{\prime 2} e^{2}\right)^{-0.5}
\end{aligned}
$$

For the reciprocal causation model, by simple algebraic manipulation of the above equalities, we find

$$
r_{\mathrm{G}}=\frac{\left(h_{\mathrm{a}}^{2} i^{\prime}+h_{\mathrm{b}}^{2} i\right)}{h_{\mathrm{a}} h_{\mathrm{b}}\left(1+i i^{\prime}\right)}, \quad r_{\mathrm{D}}=\frac{\left(d_{\mathrm{a}}^{2} i^{\prime}+d_{\mathrm{b}}^{2} i\right)}{d_{\mathrm{a}} d_{\mathrm{b}}\left(1+i i^{\prime}\right)}, \quad \text { etc. }
$$

these expressions simplifying, in the case of unidirectional causation, to $r_{\mathrm{G}}=i^{\prime} h_{\mathrm{a}} / h_{\mathrm{b}}, r_{\mathrm{D}}=i^{\prime} d_{\mathrm{a}} / d_{\mathrm{b}}$, etc. (if $A \rightarrow B$ ), or alternatively, if $B \rightarrow A, r_{\mathrm{G}}=$ $i h_{\mathrm{b}} / h_{\mathrm{a}}, r_{\mathrm{D}}=i d_{\mathrm{b}} / d_{\mathrm{a}}$, with similar expressions for $r_{\mathrm{C}}$ and $r_{\mathrm{E}}$. In other words, if the true model is the reciprocal causation model, and we fit the general bivariate model but estimate genetic and environmental parameters $h_{\mathrm{a}}$, etc., and $h_{\mathrm{b}}$, etc., together with parameters $i$ and $i$, correlations $r_{\mathrm{G}}, r_{\mathrm{D}}, r_{\mathrm{C}}$, and $r_{\mathrm{E}}$ can all be derived as functions of the other parameters, i.e., are constrained parameters. Thus the unidirectional and reciprocal causation models are submodels of the general bivariate model. In the specific case where two traits have identical modes of inheritance, i.e., $h_{\mathrm{a}}=h_{\mathrm{b}}, d_{\mathrm{a}}=d_{\mathrm{b}}, c_{\mathrm{a}}=$ $c_{\mathrm{b}}$, and $e_{\mathrm{a}}=e_{\mathrm{b}}$, we will obtain the same estimates for $r_{\mathrm{G}}$, etc., regardless of whether we assume $A \rightarrow$ $B$ or $B \rightarrow A$, confirming that in this particular instance there is no information about direction of causation. Likewise, if the direct genetic and environmental effects on each trait are identical, i.e., $h=h^{\prime}, d=d^{\prime}, c=c^{\prime}$, and $e=e^{\prime}$, we will again ob-

\begin{tabular}{|c|c|}
\hline \multicolumn{2}{|r|}{ General bivariate model } \\
\hline $\begin{array}{l}\text { Variances } \\
\text { Trait } A \\
\text { Trait } B\end{array}$ & $\begin{array}{l}\Lambda^{2}\left(h_{\mathrm{a}}^{2}+d_{\mathrm{a}}^{2}+c_{\mathrm{a}}^{2}+e_{\mathrm{a}}^{2}\right)+\theta_{\mathrm{A}} \\
\Lambda^{\prime 2}\left(h_{\mathrm{b}}^{2}+d_{\mathrm{b}}^{2}+c_{\mathrm{b}}^{2}+e_{\mathrm{b}}^{2}\right)+\theta_{\mathrm{B}}\end{array}$ \\
\hline $\begin{array}{l}\text { Covariances } \\
\qquad \begin{array}{l}A j, B j ; A k, B k \\
A j, A k \\
B j, B k \\
A j, B k ; B j, A k\end{array}\end{array}$ & $\begin{array}{l}\Lambda \Lambda^{\prime}\left(h_{\mathrm{a}} h_{\mathrm{b}} r_{\mathrm{A}}+d_{\mathrm{a}} d_{\mathrm{b}} r_{\mathrm{D}}+c_{\mathrm{a}} c_{\mathrm{b}} r_{\mathrm{C}}+e_{\mathrm{a}} e_{\mathrm{b}} r_{\mathrm{E}}\right) \\
\Lambda^{2}\left(\alpha h_{\mathrm{a}}^{2}+\delta d_{\mathrm{a}}^{2}+\beta c_{\mathrm{a}}^{2}\right) \\
\Lambda^{\prime 2}\left(\alpha h_{\mathrm{b}}^{2}+\delta d_{\mathrm{b}}^{2}+\beta c_{\mathrm{b}}^{2}\right) \\
\Lambda \Lambda^{\prime}\left(\alpha h_{\mathrm{a}} h_{\mathrm{b}} r_{\mathrm{A}}+\delta d_{\mathrm{a}} d_{\mathrm{b}} r_{\mathrm{D}}+\beta c_{\mathrm{a}} c_{\mathrm{b}} r_{\mathrm{C}}\right)\end{array}$ \\
\hline & Reciprocal causation model \\
\hline $\begin{array}{l}\text { Variances } \\
\text { Trait } A \\
\text { Trait } B\end{array}$ & $\begin{array}{l}\Lambda^{2}\left(1-i i^{\prime}\right)^{-2}\left[h^{2}+d^{2}+c^{2}+e^{2}+i^{2}\left(h^{\prime 2}+d^{\prime 2}+c^{\prime 2}+e^{\prime 2}\right)\right]+\theta_{\mathrm{A}} \\
\Lambda^{\prime 2}\left(1-i i^{\prime}\right)^{-2}\left[h^{\prime 2}+d^{\prime 2}+c^{\prime 2}+e^{\prime 2}+i^{\prime 2}\left(h^{2}+d^{2}+c^{2}+e^{2}\right)\right]+\theta_{\mathrm{B}}\end{array}$ \\
\hline $\begin{array}{l}\text { Covariances } \\
\qquad A j, B j ; A k, B k \\
A j, A k \\
B j, B k \\
A j, B k ; B j, A k\end{array}$ & $\begin{array}{l}\Lambda \Lambda^{\prime}\left(1-i i^{\prime}\right)^{-2}\left[i^{\prime}\left(h^{2}+d^{2}+c^{2}+e^{2}\right)+i\left(h^{\prime 2}+d^{\prime 2}+c^{\prime 2}+e^{\prime 2}\right)\right] \\
\Lambda^{2}\left(1-i i^{\prime}\right)^{-2}\left[\alpha\left(h^{2}+i^{2} h^{\prime 2}\right)+\delta\left(d^{2}+i^{2} d^{\prime 2}\right)+\beta\left(c^{2}+i^{2} c^{\prime 2}\right)\right] \\
\Lambda^{\prime 2}\left(1-i i^{\prime}\right)^{-2}\left[\alpha\left(h^{\prime 2}+i^{\prime 2} h^{2}\right)+\delta\left(d^{\prime 2}+i^{\prime 2}+d^{2}\right)+\beta\left(c^{\prime 2}+i^{\prime 2} c^{2}\right)\right] \\
\Lambda \Lambda^{\prime}\left(1-i i^{\prime}\right)^{-2}\left[\alpha\left(i^{\prime} h^{2}+i h^{\prime 2}\right)+\delta\left(i^{\prime} d^{2}+i d^{\prime 2}\right)+\beta\left(i^{\prime} c^{2}+i c^{\prime 2}\right)\right]\end{array}$ \\
\hline
\end{tabular}
tain the same estimates for $r_{\mathrm{G}}, r_{\mathrm{D}}, r_{\mathrm{C}}$, and $r_{\mathrm{E}}$, regardless of whether we assume $A \rightarrow B$ or $B \rightarrow A$. In general, however, this will not be the case.

Because the unidirectional and reciprocal causal models are submodels of the full bivariate model, their goodness of fit can be compared to that of the

Table I. Expected Variances and Covariances of Relatives Under General Bivariate and Reciprocal Causation Models: Parameters Are Defined in Fig. 3 and Text 
full bivariate model by likelihood-ratio chi-square test, with the numbers of degrees of freedom equal to the number of genetic and environmental correlations estimated in the bivariate model minus the number of causal paths estimated in the causal model. An important implication of this is that in order to test the reciprocal causation model against the general bivariate model, we need to have at least three "sources of variation" [in the traditional terminology of quantitative genetic (Mather and Jinks, 1971; Jinks and Fulker, 1970; Eaves, 1977)] influencing the two traits, either directly or indirectly, counting within-family environment, additive genetic effects, dominance genetic effects, and shared environment each as one source of variation. (It is not necessary that each trait be directly influenced by three sources of variation, for example, the genetic influences on $B$ may be those mediated through the causal influence of $A$ on $B$, as in Fig. 2). If there are only two "sources of variation" in the two traits, e.g., if both traits are influenced by additive genetic and within-family environmental effects, the reciprocal causation model will give the same fit to the data as the general bivariate model, and no test of the model will be available.

\section{An Important Limitation}

The fact that the reciprocal causation model is a submodel of the general bivariate model emphasizes an important difference between the inferences about causality which can be drawn using instrumental variables and the inferences which can be drawn from family data. Using an instrumental variable approach, in addition to the direct effects of $A$ on $B$ and $B$ on $A$, it is possible to estimate a residual correlation between the reciprocally interacting variables $\left(\psi_{\mathrm{AB}}\right.$ in Fig. 1). Thus it is possible to test the hypothesis that the correlation between $A$ and $B$ arises solely because of the reciprocal causal influence of $A$ on $B$ and of $B$ on $A$, plus the correlation between the instruments for $A$ and $B\left(\phi_{\mathrm{AB}}\right)$, i.e., that $\Psi_{\mathrm{AB}}=0$. In family data, in contrast, at least in the bivariate case, we are restricted to testing hypotheses of the form "the ONLY cause of the correlation between $P_{\mathrm{A}}$ and $P_{\mathrm{B}}$ is the causal influence of $P_{\mathrm{A}}$ on $P_{\mathrm{B}}$ (or the reciprocal causal influence of $P_{\mathrm{A}}$ and $P_{\mathrm{B}}$ on each other)." In what follows, we use the phrase "testing the causal hypothesis $A \rightarrow B$ (or $A \rightleftarrows B$ )" as a short-hand for these strong causal hypotheses. If $A$ and $B$ are correlated, both because of their reciprocal causal in- fluence and also because both variables are influenced by other unmeasured variables, this will cause failure of the reciprocal causation model (if the latter influences are sufficiently important). The latter case cannot be distinguished from the general bivariate model in family data on a single pair of variables but may still be tractable if our hypothesis specifies a causal chain $(A \rightarrow B \rightarrow C \rightarrow D$, etc.) with an additional contribution of other unmeasured variables to correlations between variables $A, B, C, D$, etc. The same problem is encountered in trying to draw inferences about causality from panel data, where partial determination of $A$ and $B$ by an unmeasured latent variable, $C$, can again lead to errors of inference about causality.

\section{The Problem of Measurement Error}

In general, we cannot assume that our variables are measured without error. The example of smoking status, i.e., whether or not an individual ever smoked (Heath et al., 1993), and age at death provides a rare counterinstance where measurement error effects may be minor. Under the general bivariate model, if we ignore measurement error when it is present, and if errors of measurement are uncorrelated between family members (and therefore contribute to within-family variance but not to family resemblance), this will inflate estimates of $e_{\mathrm{a}}{ }^{2}$ and $e_{\mathrm{b}}{ }^{2}$ and lead to an underestimate of $r_{\mathrm{E}}$ (Eaves and Eysenck, 1975), but other parameters will be unbiased. Similarly, in the context of the reciprocal causation and unidirectional causation submodels, ignoring measurement error will lead to inflated estimates of $e^{2}$ and $e^{\prime 2}$. However, since the expectation for the within-person (i.e., phenotypic) covariance of traits $A$ and $B\left(A_{j}\right.$ with $B_{j}$ and $A_{k}$ with $B_{k}$ in Table I) includes terms in $i^{\prime} e^{2}$ and $i e^{\prime 2}$ (see Table I), ignoring measurement error will also lead to biased estimates of all the other parameters of the direction-of-causation model.

In the case of unidirectional causal models, only a single error term is critical, namely, the error variance for trait $A$ if $A \rightarrow B$ or the error variance for trait $B$ if $B \rightarrow A$. If the direction of causation is $A \rightarrow B$ (with $i=0, i^{\prime} \neq 0$ ), then the expectation for the phenotypic covariance of $A$ and $B$ will include only the expression $i^{\prime} e^{2}$. Omitting the error term for $B$ will inflate the estimate of $e^{\prime 2}$ by the error variance for $B$, but other parameter estimates will be unbiased, provided that an error variance for trait $A$ is included in the model. Omission of 
the error variance for $A$ will, however, lead to biased parameter estimates, even if an error variance is estimated for $B$.

We reported above expectations for the genetic and environmental correlations of the general bivariate model for the case where the true model is the reciprocal causation model, for example,

$$
r_{\mathrm{E}}=\frac{\left(e_{\mathrm{a}}^{2} i^{\prime}+e_{\mathrm{b}}^{2} i\right)}{e_{\mathrm{a}} e_{\mathrm{b}}\left(1+i i^{\prime}\right)}
$$

with similar expressions for $r_{\mathrm{G}}, r_{\mathrm{D}}$, and $r_{\mathrm{C}}$. Thus even in the case where we do not have data about measurement error, it is possible to test a unidirectional causal model allowing for measurement error ("unidirectional causation with error" model), provided that we have at least three "sources of variation" influencing the two traits, either directly or indirectly. Including a single error variance in a unidirectional causation model has the effect of relaxing the equality constraint relating $r_{\mathrm{E}}$ to parameters $e_{\mathrm{a}}, e_{\mathrm{b}}$, and $i$ (or $i^{\prime}$ ). If there are three sources of variance for each trait, e.g., both trait $A$ and trait $B$ are influenced by additive gene action, shared environmental effects, and within-family environmental effects, there will remain one degree of freedom for testing the goodness of fit of each unidirectional causation with error model against the fit of the general bivariate model, which in effect is testing whether the equalities $r_{\mathrm{G}}=i^{\prime} \mathrm{h}_{\mathrm{a}} / h_{\mathrm{b}}$ and $r_{\mathrm{C}}=i^{\prime} c_{\mathrm{a}} / c_{\mathrm{b}}$ (if $A \rightarrow B$ ) or, alternatively $r_{\mathrm{G}}=$ $i h_{\mathrm{b}} / h_{\mathrm{a}}$ and $r_{\mathrm{C}}=i c_{\mathrm{b}} / c_{\mathrm{a}}$ (if $B \rightarrow A$ ) are both satisfied, and two degrees of freedom if there are four sources of variation. If there are only two sources of variation for traits $A$ and $B$, e.g., within-family environmental effects plus additive genetic effects on both traits or within-family environmental effects plus shared environmental effects on both traits, however, the general bivariate model will include only two correlations, $r_{\mathrm{E}}$ and either $r_{\mathrm{G}}$ or $r_{\mathrm{C}}$. Relaxing the constraint on $r_{E}$, by estimating an error variance for one variable, will be equivalent to fitting the general bivariate model. If there are only two sources of variation, therefore, the two "unidirectional causation plus measurement error" models will, in general, give identically good fits to the data and will give the same fit as the general bivariate model. In this case we can proceed to test causal hypotheses only if we know that both traits are measured without error or if we have additional information about error variances for the two traits and can exploit this in a multiple indicator causal model.
In the case of the reciprocal causation model, within-family environmental variances for both traits occur in the expectation for the within-person covariance of the two traits. If no other data about error variances are available, estimation of an error variance for one trait will relax the equality constraint on $r_{\mathrm{E}}$. Regardless of how many sources of variation are influencing the two traits, no further information is available to estimate an error variance for the second trait. Thus it will be possible to test reciprocal causation models only if we are prepared to assume that the error variances for the two traits are either zero or equal in absolute magnitude [it appears that relatively small differences in the magnitude of the error variances for $A$ and $B$ will produce only a minor bias to estimates of other parameters, if the error variances are assumed equal (Neale and Cardon, 1992)] or if additional information is available to estimate the effects of measurement error for each trait, for example, by fitting a multiple indicator model. If error variances can be assumed to be zero, the reciprocal causation model will be just identified if there are only two sources of variation, and at least three sources of variability will be required in order to permit a likelihood-ratio chi-square test, on one degree of freedom, against the general bivariate model. If error variances are nonzero but can be assumed to be equal, four sources of variability will be required to permit a test of the reciprocal causation plus error model against the general bivariate model. The sequence of causal models that can be tested and degrees of freedom for testing each model against the general bivariate model are summarized in Table III.

\section{Multiple Indicator and Multivariate Genetic Models}

In studies of samples of unrelated individuals, generalization of the instrumental variable or panel models to allow for multiple measures ("indicators") of traits $A$ and $B$ and their instruments is comparatively straightforward and is illustrated in standard texts on structural equation modeling (e.g., Bollen, 1989). The use of multiple indicator variables in family studies enjoys the same advantages as in the more traditional approaches to testing causal hypotheses, i.e., it allows the formulation of explicit assumptions about measurement error and testing of at least some of these assumptions; for example, depending upon how many indicator var- 
iables are assessed, it may be possible to allow for correlated errors of measurement between certain indicators (Costner and Schoenberg, 1973). In family data, the use of multiple indicator variables also makes it possible to allow for correlated errors of measurement between family members for each indicator variable (Martin and Eaves, 1977). We have noted in the previous section that multiple indicator data are needed to allow estimation of reciprocal causal effects, except in cases where error variances are known precisely from external sources. In this section, therefore, we first present a multiple indicator generalization of the direction of causation model for family data and then compare this model to less restrictive multivariate genetic models.

\section{Multiple Indicator DOC Model}

Figure 4 represents a simple extension of the direction-of-causation model in Fig. 3, allowing for multiple indicators of traits $A$ and $B$. The path diagram illustrated in Fig. 4 assumes that we have three observed variables which are hypothesized to

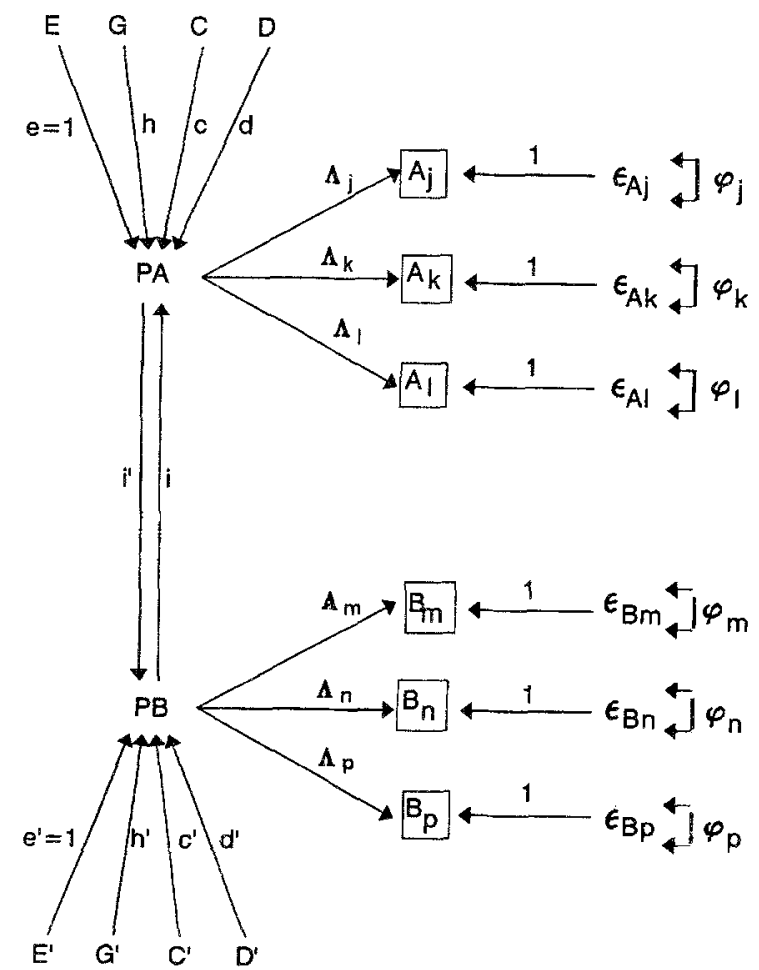

Fig. 4. Multiple indicator direction-of-causation path model. See Fig. 3 and text for definition of variables and parameters. be measures of trait $A$ and three observed variables which are hypothesized to be measures of trait $B$. Structural equations for $P_{\mathrm{A}}$ and $P_{\mathrm{B}}$ will remain unchanged, but now we will have, in addition, for the $m$ th relative,

$$
\begin{array}{ll}
Y_{\mathrm{Ajm}}=\lambda_{\mathrm{A} j} P_{\mathrm{A} m}+\epsilon_{\mathrm{Aj} m} & (j=1,3) \\
Y \mathrm{~B}_{k m}=\lambda_{\mathrm{B} k}^{\prime} P_{\mathrm{B} m}+\epsilon_{\mathrm{B} k m} & (k=4,6)
\end{array}
$$

In the terminology of factor analysis (Harman, 1976), the parameters $\lambda_{\mathrm{Aj}}$ and $\lambda_{\mathrm{B} k}^{\prime}$ are common factor loadings of manifest variables $A_{j}$ and $B_{k}$ (i.e., the $j$ th and $k$ th indicator variables for traits $A$ and $B$ ) on latent factors $P A$ and $P B$, respectively. The terms $\epsilon_{\mathrm{Ajm}}$ and $\epsilon_{\mathrm{B} k m}$ will include both "specific factor" effects and measurement error effects. We may further decompose $\epsilon_{\mathrm{A} j m}$ and $\epsilon_{\mathrm{B} k m}$ into variable-specific genetic and environmental effects, e.g.,

$$
\epsilon_{\mathrm{A} j}=h_{j} G_{j m}+d_{j} D_{j m}+c_{j} C_{j m}+e_{j} E_{j m}
$$

where $G_{j m}, D_{j m} C_{j m}$ and $E_{j m}$ denote additive and dominance genetic and shared and within-family environmental effects specific to the $j$ th indicator variable, i.e., which contribute to its variance but not to its covariance with other variables, and the term $E_{j m}$ will include measurement error (Eaves, 1977; Martin and Eaves, 1977). Alternatively, and less parsimoniously, we may allow for correlations between relatives for the $\epsilon$ 's which will differ for different classes of relatives. To identify the model, we have fixed $e=e^{\prime}=1$ in Fig. 4, but note that alternative constraints, such as fixing the total variances of underlying traits $P_{\mathrm{A}}$ and $P_{\mathrm{B}}$ to unity, could be applied instead.

\section{Multivariate Genetic Model}

A general "two-factor" multivariate genetic model (Eaves, 1977; Martin and Eaves, 1977; Heath et al., 1989a, b) provides one baseline against which the fit of the multiple indicator direction-of-causation model can be compared. The general multivariate genetic model, like the general bivariate model, makes no assumptions about direction of causation. A generalization of conventional behavioral genetic (Jinks and Fulker, 1970) and factor (Harman, 1976) models, the general multivariate genetic model estimates orthogonal common and specific additive and dominance genetic and shared and within-family environmental factors (Eaves, 1977; Martin and Eaves, 1977; Heath et al., 1989a). In terms of structural equations, under the general 
model we will have (omitting subscripts for relatives)

$$
\begin{array}{r}
Y_{\mathrm{A} j}=h_{j \mathrm{a}} G_{\mathrm{a}}+h_{j \mathrm{~b}} G_{\mathrm{b}}+d_{j \mathrm{a}} D_{\mathrm{a}}+d_{j \mathrm{~b}} D_{\mathrm{b}}+c_{j \mathrm{a}} C_{\mathrm{a}} \\
+c_{j \mathrm{~b}} C_{\mathrm{b}}+e_{j \mathrm{a}} E_{\mathrm{a}}+e_{j \mathrm{~b}} E_{\mathrm{b}}+\epsilon_{\mathrm{A} j}
\end{array}
$$

where $G_{\mathrm{a}}$ and $G_{\mathrm{b}}$ denote the first and second additive genetic common factors, $D_{\mathrm{a}}$ and $D_{\mathrm{b}}$ the first and second dominance genetic common factors, and so on; $h_{j a} h_{j b}, d_{j a}, d_{j b}$, etc., are the additive and dominance genetic loadings of the $j$ th item on the first and second additive and dominance genetic common factors; and $\epsilon_{\mathrm{A} j}$ denotes the sum of the specific factor and measurement error effects for the $j$ th item. The issue of factor rotation which arises in conventional factor analysis (e.g., Harman, 1976) will apply equally to factors estimated under the multivariate genetic model, except that factors corresponding to different sources of variability cannot be rotated jointly, i.e., we must rotate separately additive genetic common factors, dominance genetic common factors, shared environmental common factors, and within-family environmental common factors. Covariances of the genetic and environmental factors between relatives may be expressed in terms of constants $\alpha$ and $\delta$ (which depend upon genetic relatedness) and $\beta$ (which depends upon rearing experience), as before, with the assumption of orthogonal genetic and environmental factors implying no cross-covariances between the first additive genetic factor in one relative and the second additive genetic factor in the second relative, etc. In terms of the parameters of the general multivariate genetic model, the expectation for the variance of observed variable $Y_{\mathrm{A} j}$ will be

$$
\begin{aligned}
\operatorname{Var}\left(Y_{\mathrm{A} j}\right)=h_{j \mathrm{a}}^{2}+h_{j \mathrm{~b}}^{2}+h_{j}^{2}+d_{j \mathrm{a}}^{2}+d_{j \mathrm{~b}}^{2}+d_{j}^{2} \\
+c_{j \mathrm{a}}^{2}+c_{j \mathrm{~b}}^{2}+c_{j}^{2}+e_{j \mathrm{a}}^{2}+e_{j \mathrm{~b}}^{2}+e_{j}^{2}
\end{aligned}
$$

the expectation for the within-person covariance of observed variables $Y_{\mathrm{A} j}$ and $Y_{\mathrm{A} k}$ will be

$$
\begin{aligned}
\operatorname{Cov}\left(Y_{\mathrm{A} j} Y_{\mathrm{Ak}}\right)= & h_{j \mathrm{a}} h_{k \mathrm{a}}+h_{j \mathrm{~b}} h_{k \mathrm{~b}}+d_{j \mathrm{a}} d_{k \mathrm{a}}+d_{j \mathrm{~b}} d_{k \mathrm{~b}} \\
& +c_{j \mathrm{a}} c_{k \mathrm{a}}+c_{j \mathrm{~b}} c_{k \mathrm{~b}}+e_{j \mathrm{a}} e_{k \mathrm{a}}+e_{j \mathrm{~b}} e_{k \mathrm{~b}}
\end{aligned}
$$

the expectation for the covariance between the $m$ th and the $n$th relatives for $Y_{\mathrm{Aj}}$ will be

$$
\begin{aligned}
& \operatorname{Cov}\left(Y_{\mathrm{A} j m} Y_{\mathrm{Ajn}}\right)=\alpha\left(h_{j \mathrm{a}}^{2}+h_{j \mathrm{~b}}^{2}+h_{j}^{2}\right) \\
& +\delta\left(d_{j \mathrm{a}}^{2}+d_{j \mathrm{~b}}^{2}+d_{j}^{2}\right)+\beta\left(c_{j \mathrm{a}}^{2}+c_{j \mathrm{~b}}^{2}+c_{j}^{2}\right)
\end{aligned}
$$

and the expectation for the covariance between $Y_{\mathrm{A} j}$ measured in relative $m$ and $Y_{\mathrm{A} k}$ measured in relative $n$ will be

$$
\begin{aligned}
& \operatorname{Cov}\left(Y_{\mathrm{A} j m} Y_{\mathrm{A} j n}\right)=\alpha\left(h_{j \mathrm{a}} h_{k \mathrm{a}}+h_{j \mathrm{~b}} h_{k \mathrm{~b}}\right) \\
& \quad+\delta\left(d_{j \mathrm{a}} d_{k \mathrm{a}}+d_{j \mathrm{~b}} d_{k \mathrm{~b}}\right)+\beta\left(c_{j \mathrm{a}} c_{k \mathrm{a}}+c_{j \mathrm{~b}} c_{k \mathrm{~b}}\right)
\end{aligned}
$$

As in the single indicator case, we also give (in Table II) expressions for the parameters of the general multivariate genetic model in terms of the parameters of the multiple indicator reciprocal causation model, which will apply when the latter is the "true" model.

The general multivariate genetic model imposes no constraints on the genetic and environmental common factor structures; it does not constrain the pattern of genetic factor loadings to bear any relationship to the pattern of environmental factor loadings. A more restrictive version of this model, which has been variously described as the "psychometric" (McArdle and Goldsmith, 1990), "common pathway" (Kendler et al., 1987), or "latent phenotype" (Heath et al., 1989a, b) model, while still making no assumptions about direction of causation, imposes the constraint that genetic and shared environmental loadings on each factor differ from the corresponding within-family environmental factor laodings only by a scale factor (Martin and Eaves, 1977). This is the pattern that we would expect to observe if indicator variables load on "phenotypic" factors (e.g., personality factors), which in turn are determined by orthogonal genetic and environmental effects. In Table II we present common factor loadings, in the form of additive genetic, shared environmental, and withinfamily environmental factor pattern matrices, for the general multivariate and psychometric genetic models. As in the case of the multiple indicator direction-of-causation model, the psychometric model may be identified by fixing $e=e^{\prime}=1$ or by constraining the variances of the underlying phenotypic factors, $P A$ and $P B$, to unity. In contrast to the general multivariate model, factor loadings under the psychometric model cannot in general be rotated (e.g., Hewitt, unpublished): the constraint that the genetic and environmental effects $\left(G\right.$ or $G^{\prime}$, $C$ or $C^{\prime}$, etc.) on intervening latent phenotypes $P A$ and $P B$ are uncorrelated identifies a unique factor solution. Finally, we may note that by combining the general bivariate model in Fig. 3 (with $i=\mathrm{i}^{\prime}=0$ ) with the multiple indicator variable component of the model in Fig. 4, we may also derive a correlated factors version of the psychometric model (McArdle and Goldsmith, 1990). Under this model, loadings of indicator variables for trait $P A$ on genetic and environmental common factors II, and 
Table II. Genetic and Environmental Factor Pattern Matrices Under (a) General Multivariate Genetic and (b) Multiple Indicator Direction-of-Causation Models ${ }^{a}$

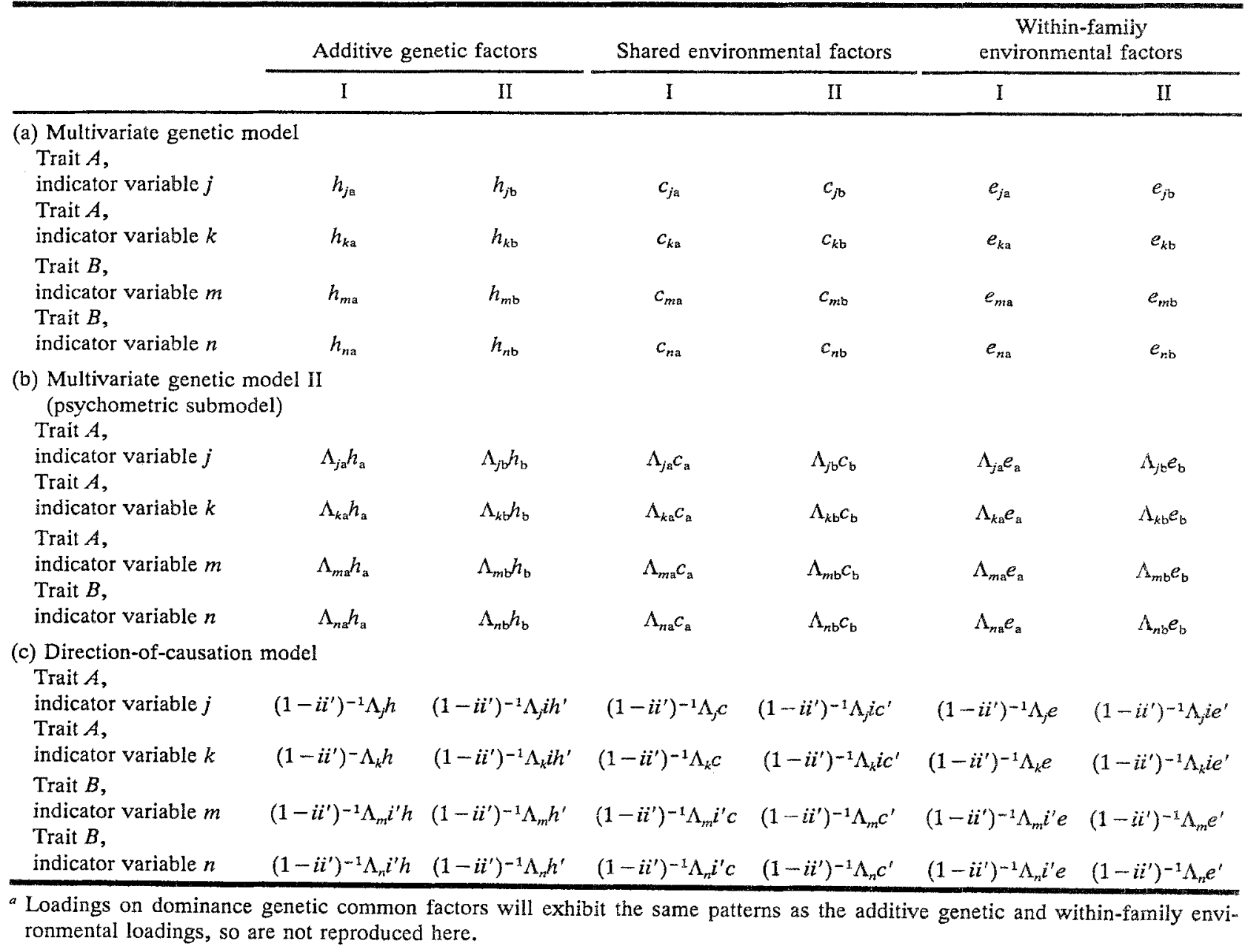

loadings of indicator variables for trait $B$ on genetic and environmental common factors $I$, are fixed to zero, but correlations $r_{\mathrm{A}}, r_{\mathrm{D}}, r_{\mathrm{C}}$, and $r_{\mathrm{E}}$ are estimated between first and second additive genetic, first and second dominance genetic common factors, etc.

\section{Relationships Between Models}

The same algebra which we used to show that the reciprocal causation model is a submodel of the general bivariate genetic model in the single indicator case applies equally when we compare the multiple indicator direction of causation model and the correlated factors psychometric model. However, if we consider the factor pattern matrices pre- dicted under the direction-of-causation model (also given in Table II), then we see that this model is also a submodel of the less restrictive psychometric model, as well as of the general multivariate genetic model. Comparing factor loadings under the psychometric model to those under the general multivariate genetic model, genetic and shared environmental common factor loadings on the first and second common factors are $h / e$ and $c / e$ and $h^{\prime} /$ $e^{\prime}$ and $c^{\prime} / e^{\prime}$ times the loadings on the first and second within-family environmental common factors, respectively. In addition to this constraint, under the multiple indicator direction of causation model, for indicator variables for trait $A$, loadings on the second common within-family environmental factor differ from those on the first common factor by 
a factor of $\left(i e^{\prime} / e\right)$, while for indicator variables for trait $B$, loadings on the first common within-family environmental factor differ from those on the second common factor by a constant multiple $\left(i^{\prime} e / e^{\prime}\right)$, with similar constraints applying to genetic and shared environmental common factor loadings. As a submodel of the psychometric model, the multiple indicator direction-of-causation model will also define a unique factor solution. Expectations for the variances and covariances of relatives under the psychometric and multiple indicator direction-ofcausation models may be derived from those for the general multivariate genetic model by making the appropriate substitutions for common factor loadings from Table II; specific factor effects will be parameterized identically under these models.

\section{Measurement Error}

From these considerations we can see how the use of multiple indicator variables will reduce the problem of measurement error, including correlated errors of measurement, in testing causal hypotheses. If measurement error is variable specific, application of the multiple indicator direction-ofcausation model to cross-sectional family data will provide statistical tests of the goodness of fit of the reciprocal causation model $(A \rightleftarrows B)$ compared to correlated factors, psychometric, or general multivariate genetic models, provided that there are at least three sources of variability, and also tests of the unidirectional causal models $(A \rightarrow B$ or $B \rightarrow$ $A$ ), even when there are only two sources of variation (e.g., additive genetic plus within-family environmental effects) in $A$ and $B$. If there are only two sources of variability, the reciprocal causation model will be confounded with the correlated psychometric factors model, the same result that we noted in the single indicator case. Measurement error correlations for the same indicator variable between family members will bias estimates of the variable-specific genetic and environmental parameters but will not affect estimates of common factor loadings and, hence, will not bias inferences about direction of causation. If measurement errors are correlated across indicator variables, but the crossvariable error correlations between family members are zero, it will still be possible to test the reciprocal and unidirectional causal models if there are at least three sources of variability (or four in the case of the reciprocal causation model). In this case, instead of constraining additive and dominance and shared environmental common factor loadings to be multiple of within-family environmental common factor loadings, we will estimate, say, dominance genetic and shared environmental common factor loadings to be multiples of the additive genetic common factor loadings but impose no constraints on the within-family environmental common factor loadings. Finally, if there are correlations between measurement errors across different indicator variables between family members, as well as within persons, then we may be able to incorporate these in our model, following the approach of Costner and Schoenberg (1973), provided that we have assessed a sufficiently large number of indicator variables and that the number of indicator variables for which such cross-indicator familial correlations occur is small.

\section{Hypothesis Testing}

Table III summarizes the series of models which may be tested using single indicator variable and multiple indicator variable data, together with the degrees of freedom for the likelihood-ratio chi-square test of each submodel against the most general model. In the case of multiple indicator variable models, many of these degrees of freedom are testing aspects of the measurement model relating the indicator variables to the underlying traits $A$ and $B$. Thus, even if a unidirectional or reciprocal causation model does not give a significantly worse fit than the general multivariate genetic model, its fit must still be compared to that of the correlated factors psychometric model. The degrees of freedom for the latter comparison will be the same as for the corresponding comparison, in the single indicator case, of the general bivariate model and the causal submodel, with no measurement error (since errors of measurement are included at the level of the indicator variable in the multiple indicator variable case).

Models may also be simplified by dropping out sources of variability for each trait, so that, in principle, for each of the models in Table III, submodels could be fitted which assume (i) no genetic effects, (ii) no shared environmental effects, (iii) no dominance effects, or (iv) no dominance or shared environmental effects. In practice, the number of models which must be fitted is rather less than this, since evidence that a source of variability must be retained in the most general model indicates that it will be needed in all submodels. A source of var- 
Table III. Nested Models to Be Compared When Testing Direction-of-Causation Models $^{a}$

\begin{tabular}{|c|c|}
\hline & $\begin{array}{l}\text { Degrees of freedom } \\
\text { vs. } \\
\text { general model }\end{array}$ \\
\hline \multicolumn{2}{|l|}{ Single indicator variable models } \\
\hline 1. $A \rightarrow B$, no measurement error & $s-1$ \\
\hline 2. $A \rightarrow B$, measurement error in $A$ & $s-2$ \\
\hline 3. $B \rightarrow A$, no measurement error & $s-1$ \\
\hline$B \rightarrow A$, measurement error in $B$ & $s-2$ \\
\hline 5. $A \rightleftarrows B$, no measurement error & $s-2$ \\
\hline 6. $A \rightleftarrows B$, identical error variances for $A, B$ & $s-3$ \\
\hline 7. General bivariate model & - \\
\hline \multicolumn{2}{|l|}{ Multiple indicator variable models } \\
\hline 1. $A \rightarrow B$ & $2(2 k-1)(s-1)+2 k-1$ \\
\hline$B \rightarrow A$ & $2(2 k-1)(s-1)+2 k-1$ \\
\hline$A \rightleftarrows B$ & $2(2 k-1)(s-1)+2 k-2$ \\
\hline Correlated factors psychometric model & $2(2 k-1)(s-1)+2 k-s$ \\
\hline Orthogonal psychometric model & $2(2 k-1)(s-1$ \\
\hline 6. General multivariate genetic model & - \\
\hline \multicolumn{2}{|c|}{$\begin{array}{l}s \text { denotes the number of sources of variability, counting additive genetic, dominanc } \\
\text { genetic, shared environment, and within-family environment effects each as one source } \\
k \text { denotes the number of indicator variables per trait, with the total number of manifes } \\
\text { variables }=2 k \text {. }\end{array}$} \\
\hline \multicolumn{2}{|c|}{$\begin{array}{l}{ }^{b} \text { Degrees of freedom for likelihood-ratio chi-square test versus general model. } \\
\text { All models estimate variable-specific genetic and environmental factors. }\end{array}$} \\
\hline
\end{tabular}

iability which is nonsignificant in the most general model, however, may nonetheless be significant in a submodel. Models which estimate dominance genetic effects but not additive genetic effects have no sensible biological interpretation (Mather and Jinks, 1971) and, so, are not fitted. Although, in principle, it is possible to fit multiple indicator models which include no common factor within-family environmental effects, these imply (in the case of the psychometric and direction-of-causation models) completely heritable traits, an unlikely result for behavioral variables.

Choice of a particular experimental design may limit the ability of a study to resolve certain sources of variability, which in turn may influence the range of submodels which can be compared. These issues can be particularly tricky in the multivariate case. Most applications of direction-of-causation models, for example, have used data on monozygotic and dizygotic twin pairs reared together, the "classical twin design," in which the effects of genetic dominance and shared environment are confounded (Eaves, 1969, 1970; Martin et al., 1978): genetic dominance (in the absence of shared environmental effects) generates dizygotic twin covariances which are less than one-half the monozygotic covariances, while shared environmental effects (in the absence of genetic dominance) will produce dizygotic twin covariances which are greater than one-half the monozygotic covariances. Thus the effects of genetic dominance and shared environment on the same trait cannot be resolved using only data on twin pairs reared together, except in the case where there is no genetic variance at all (implying identical monozygotic and dizygotic covariances). A single indicator direction-of-causation model in which there are direct additive and dominance genetic effects on one trait $(A)$ and direct shared environmental effects on the second trait $(B)$, with unidirectional causation or reciprocal causation between the two traits (see Fig. 2), is identified in classical twin data. The general bivariate model, however, would require estimation of correlated dominance and shared environmental effects for both traits and, so, will not be identified in classical twin data! In this example, therefore, the appropriate likelihood-ratio comparison of the reciprocal causation model to the most general model is not available. The two unidirectional models likewise cannot be tested against the general bivariate model but are both submodels 
of the reciprocal causation model, so that likelihood-ratio comparisons with that model can be made. For the same example, but assuming multiple indicator variables, the correlated factors psychometric model will not be identified, by the same reasoning that applies to the general bivariate model with single indicator variables. The orthogonal factors psychometric model, with one factor allowing for genetic dominance and the second for shared environmental effects, will be identified and, thus, permits a test of the reciprocal causation model as well as of the two unidirectional causal models. Using multiple relationships to ensure that the major sources of variability are unconfounded (Fulker, 1982; Heath, 1983; Heath et al., 1985) will help avoid such complications.

\section{Power Analyses}

Demonstration that it is feasible, in principle, to test strong causal hypotheses using cross-sectional family data need not imply that it is feasible in practice. We present in this section details of power analyses using the noncentral chi-square distribution (Martin et al., 1978; Heath and Eaves, 1985; Heath et al., 1985; Matsueda and Bielby, 1986; Satorra and Saris, 1985), which were conducted for a limited range of parameter values, and considered only the power of the most widely used experimental design, the classical twin design. These suffice to demonstrate the conditions under which resolution of hypotheses about direction of causation is likely to be most feasible. For a given true model (e.g., $A \rightarrow B$ ), and given population parameter values, we generated numerical values for the expected covariances for monozygotic and dizygotic twin pairs reared together. Genetic and environmental parameter values were chosen to give total variances for $P A$ and $P B$, in the absence of any causaul influence of $P A$ on $P B$, or vice versa, of unity. (We have taken this approach, rather than standardizing parameter estimates after allowing for the causal effects of $B$ on $A$, or vice versa, since it simplifies presentation of results for the case of reciprocal interaction: see below.) A false model (e.g., $B \rightarrow A$ or $B \rightarrow A$ with measurement error in $B$ ) was fitted to the data, assuming equal numbers of monozygotic and dizygotic twin pairs and an arbitrary total sample size, $N$, and the goodness-of-fit chi-square, $C$, was recorded. Since the true model, and all models of which the true model is a submodel, would give a perfect fit to the observed data, the statistic $C$ is also a likelihood-ratio chisquare for testing the false model against a more general model of which both the true and the false models are submodels, with degrees of freedom, $x$, equal to the number of parameters of the more general model which have been fixed to zero or unity under the false model. The sample sizes required for $80 \%$ probability of rejecting the false model, at the $5 \%$ significance level, given the population parameters used under the true model, were compared (see Martin et al., 1978; Satorra and Saris, 1985) as

\section{$N C^{\prime} / C$}

where $C^{\prime}$ is the noncentral chi-square parameter $C_{(0.05,80, x)}$ obtained from the table of noncentral chisquares (Pearson and Hartley, 1972).

In our analyses of statistical power, we considered first the case where only a single indicator variable is used for each trait. We considered both the case where both traits are measured without error and then the more realistic case where $20 \%$ of the observed variance in trait $A$, and also $20 \%$ of the observed variance in trait $B$, is attributable to error variance. In model-fitting, however, in every case the models fitted included a false unidirectional model with measurement error, since in some cases this required larger sample sizes to reject than the false model assuming no measurement error (e.g., cases 1 and 2 when the true model was $A \rightarrow B$ ). We examined four cases: (1) direct effects of additive and dominance gene action on trait $A$ and of shared environment on trait $B$ but with no direct shared environmental influence on trait $A$ and no direct genetic influence on trait $B$; (2) direct additive genetic effects only on trait $A$ and direct shared environmental effects only on trait $B$; (3) direct additive genetic and dominance effects on trait $A$ and direct additive genetic and shared environmental effects on trait $B$; and (4) direct additive genetic and shared environmental effects on both traits $A$ and $B$, with genetic effects having a relatively greater impact on trait $A$, and shared environmental effects on trait $B$. In each case we compared the statistical power when nonshared environment is accounting for $25 \%$ of the variance and also, in the case of true models without measurement error, $50 \%$ of the variance.

Illustrative power analyses were also conducted under the multiple indicator model, assuming three indicator variables for each trait. These used the same sets of parameters values as under the measurement error analyses and assumed that 
measurement error variance is the only source of specific factor variance for the indicator variables, accounting for only $20 \%$ of the variance. These are very strong assumptions, since empirically it has usually been found that variable-specific genetic and shared environmental influences are also found (e.g., Martin and Eaves, 1977; Eaves et al., 1989).

\section{RESULTS}

Table IV summarizes the number of twin pairs required, assuming equal numbers of monozygotic and dizygotic twin pairs reared together are used in a study, for $80 \%$ power of rejecting a false unidirectional causal hypothesis, at the 5\% significance level, when the true population model is the alternative unidirectional causal model. It demonstrates very clearly that the power of the twin design to falsify unidirectional causal models is greatest when the two correlated traits have very different modes of inheritance. To provide some perspective for the sample sizes reported in Table IV, a sample size of $200-500$ twin pairs is achievable for a laboratory study in which twin pairs are participating in an experimental protocol (e.g., Martin et al., 1985a, b; Scheiken et al., 1989), 1000 or more twin pairs in an interview-based study (e.g., Kendler et al., 1991), and as many as 5000 or more twin pairs in an mailed questionnaire survey (e.g., Eaves et al., 1992; Eisen et al., 1991). We consider first cases where measurement error is negligible. The worst case is that where both traits are influenced by both additive genetic and shared environmental effects, albeit to differing degrees (case I.4). For this case our ability to reject the false unidirectional causal model even in questionnaire survey data depends upon within-family environmental effects having a relatively modest impact $\left(e^{2}=e^{\prime 2}=0.25\right)$ and the causal influence being at least moderately strong $\left(1>0.25\right.$ or $\left.i^{\prime}>0.25\right)$ or, if there is a stronger influence of within-family environmental variance $\left(e^{2}=e^{\prime 2}=0.5\right)$, requires that the causal influence be very strong indeed $\left(i>0.5\right.$ or $\left.i^{\prime}>0.5\right)$. In the first two cases, where there are no direct genetic influences on trait $B$, and direct genetic but no direct shared environmental effects on trait $A$, if withinfamily environment effects are weak, required sample sizes are within a range that can be achieved in laboratory studies if $i$ or $i^{\prime} \geq 0.3$ and are certainly achievable in interview studies if $i$ or $i^{\prime} \geq 0.15$. In the third case, where there are direct additive genetic effects on both traits, but direct nonadditive (dominance) effects on trait $A$ and direct shared environmental effects on trait $B$, sample sizes are within the feasible range for interview-based studies if $e^{2}=e^{\prime 2}=0.25$ and $i \geq 0.25$ or $i^{\prime} \geq 0.25$, or if $e^{2}=e^{\prime 2}=0.5$ and $i \geq 0.4$ or $i^{\prime} \geq 0.4$. In all cases, increasing the proportion of variance accounted for by within-family environment (and therefore decreasing twin pair resemblance) greatly increases the required sample sizes.

Once we allow for levels of measurement error that are realistic for many psychometric traits (or even optimistic!), i.e., with $20 \%$ of the observed variance in each trait being explained by measurement error, sample sizes move beyond the level that would be feasible for laboratory-based experimental studies. When the two traits have essentially similar modes of inheritance (case II.4), but with differences in the relative magnitude of genetic and shared environmental effects, even for large-scale questionnaire surveys the required sample sizes are impractically large unless the unidirectional causal influence is very large $\left(i \geq 0.4\right.$ or $i^{\prime} \geq 0.4$ ). However, when modes of inheritance are somewhat different (cases II.1-II.3), sample sizes are well within the feasible range for mailed questionnaire studies and, at values of $i$ or $i^{\prime}$ $\geq 0.3$ (cases II.1 and II.2) or 0.4 (case II.3), are feasible for interview-based studies.

It is instructive to consider the case where the true model involves reciprocal causation between the two traits and where, under the true model, the two traits are measured without error. It turns out that for the parameters given in Table IV for the case of no measurement error, which were chosen so that $\left(e^{2}+h^{2}+d^{2}+c^{2}\right)=1$ and $\left(e^{\prime 2}+h^{\prime 2}\right.$ $\left.+c^{\prime 2}\right)=1$, the goodness-of fit chi-square for rejecting the simple $A \rightarrow B$ model, or the same model allowing for measurement error, does not depend upon the value of $i^{\prime}$ and is the same as the chisquare for rejecting the simple $A \rightarrow B$ model when the true model is $B \rightarrow A$; and likewise, the goodness-of-fit chi-square for rejecting the $B \rightarrow A$ model does not depend upon the value of $i$ and is the same as the chi-square for rejecting the simple $B \rightarrow A$ model when the true model is $A \rightarrow B$. Thus the sample sizes required for rejecting either simple unidirectional causation model, allowing for the possibility of measurement error, can be read directly from Table IV; for example, under case I.1, with $e^{2}=0.25$ and $i=i^{\prime}=0.3,313$ pairs are required to reject the false unidirectional model $A \rightarrow B$, and 248 pairs are required to reject the unidirectional model $B \rightarrow A$. 


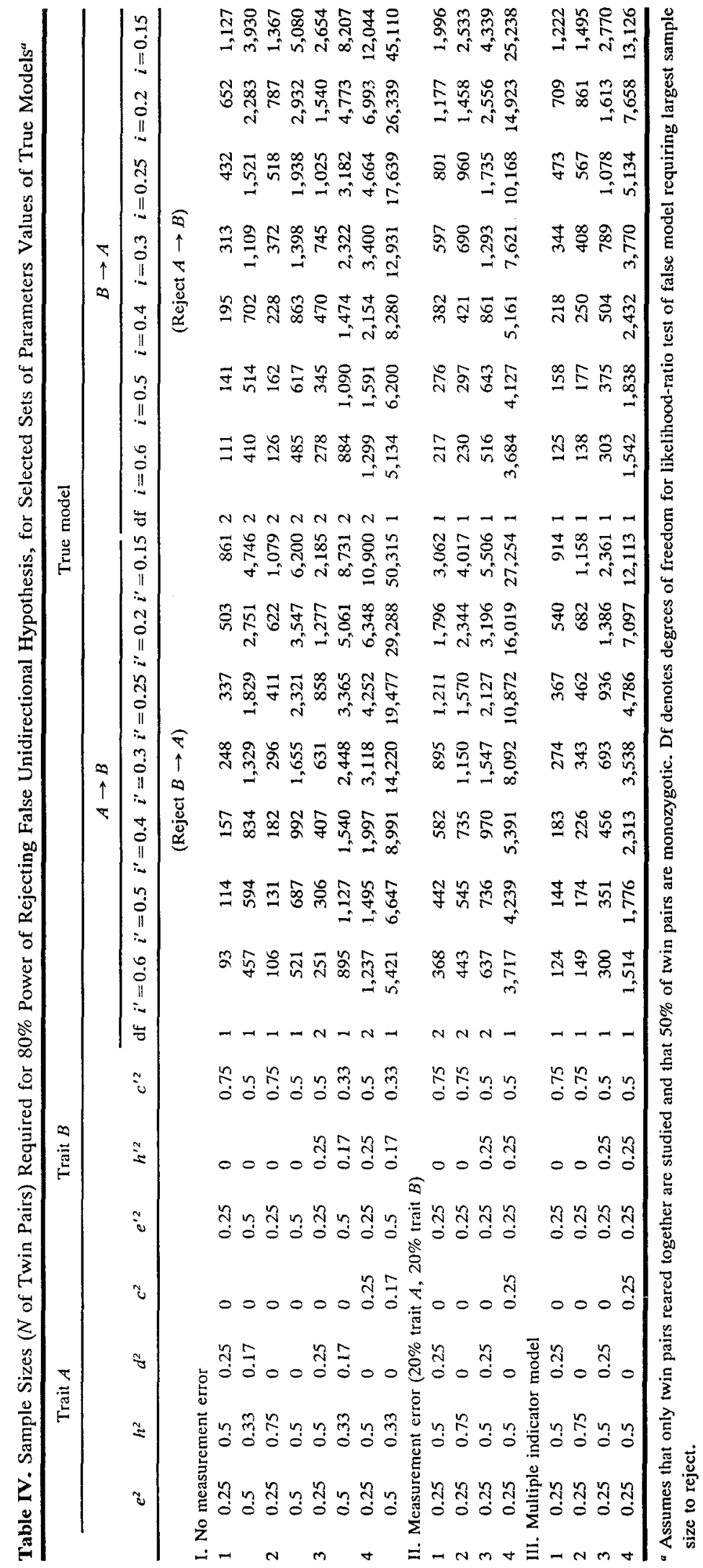


Also given in Table IV are illustrative power analyses under the multiple indicator model (III.1III.4). These show that, under the strong assumptions used here, the power of the multiple indicator model in the presence of moderate levels of measurement error is not much reduced compared to that of the single indicator model under the idealized conditions of no measurement error. These analyses again confirm the finding that direction of causation analyses of two correlated traits have a relatively high power of resolving unidirectional causal hypotheses if their modes of inheritance are very different, even if their association is relatively weak, but a rather low power if their modes of inheritance are rather similar, even when their association is rather strong. They also confirm the importance of using multiple indicator variables.

\section{DISCUSSION}

There are many cases in the behavioral sciences where the direction of causation underlying an association between two variables is uncertain, and the possibility of a reciprocal causal influence of each variable on the other must be considered a possibility. Despite the theoretical appeal of methods using instrumental variables (Kessler, 1983) or panel data (e.g., Duncan, 1969; Kenny, 1975), practical application of these methods is often problematic. We have explored the conditions under which even cross-sectional data on family members (for example, monozygotic and dizygotic twin pairs or adoptees and their biological and adoptive parents and siblings) may be used to test hypotheses about direction of causation. When two correlated traits have somewhat different modes of inheritance, as would be the case if one trait is strongly influenced by family environmental effects and the other by genetic effects, the cross-correlations between one trait in one relative and the second trait in the second relative may provide important information about direction of causation. However, in contrast to instrumental variable methods, it is possible, when using family data on pairs of variables, only to test strong hypotheses of the form " $A$ and $B$ are correlated solely because of the causal influence of $A$ on $B$ (and/or of $B$ on $A$ )." When applied to pairs of variables, this approach does not require that there be no other unmeasured causes of $B$ than $A$, or vice versa; but it does require that there are no other unmeasured causes of $A$ that are also causes of $B$. Thus, to give a concrete example, these methods can be used to falsify the hypotheses that the correlation between retrospective self-reports of early rejection by parents and history of depression (e.g., Parker, 1983, 1990) arises (i) solely because of a recall bias in those with a history of depression (depression $\rightarrow$ parental rejection) or, alternatively, (ii) solely because parental rejection is a risk factor for depression (rejection $\rightarrow$ depression); but rejection of these two simple hypotheses does not preclude the possibility that, for example, parental rejection is a cause of depression, and in addition, certain temperamental and personality traits predispose both to parental rejection and to depression. This restriction no longer applies if our model consists of a causal chain or simplex of the form $A \rightarrow B \rightarrow C \rightarrow D$, with at least four variables (e.g., Treloaar and Martin, unpublished), but cases of such precise a priori hypotheses are rare in the behavioral sciences!

Although we are limited to testing strong causal hypotheses using family data, even this limited capability may be very useful. Strong causal hypotheses are commonly used when assessing (or asserting) the importance of risk factors for psychopathology, and the ability to demonstrate that an association is in part "spurious" (Kenny, 1975; Kessler et al., 1992), i.e., not due solely to the causal influence of the risk factor on risk of psychopathology, is an important advantage. Retrospective reports of early experiences (Yarrow, 1963) or even ratings of an individual's contemporary environment (Brown and Harris, 1978) may be expected to show spurious associations with psychopathology, arising through such mechanisms as the impact of psychopathology on selective recall or the biasing influence of knowledge of an individual's psychiatric history on ratings by other informants. Prospective longitudinal studies, using sample sizes sufficiently large to ensure adequate numbers of cases of psychopathology in adulthood, are rarely economically feasible, and their interpretation is rendered problematic by the cumulative effects of attrition. Such longitudinal studies are also fraught with eithical difficulties, since the moral responsibility to provide information about treatment options (e.g., if cases of untreated hyperactivity or of adolescent substance abuse are detected) may interfere with the scientific interest of obtaining natural history data in untreated subjects. The capability of using retrospective data on genetically informative family members to reject the extreme hypotheses of (i) no spuriousness (e.g., early experience $\rightarrow$ psychopathology) and (ii) 
a purely spurious relationship (e.g., psychopathology $\rightarrow$ perceived environment) is therefore a very important one.

The conditions under which family data are informative about direction of causation, at least for studies using realistic sample sizes, are quite restricted. In the case of pairs of variables, there is no information about direction of causation if the two variables have identical patterns of familial transmission, i.e., if additive and nonadditive genetic and shared and within-family environmental variances are identical for each trait. If only single measures (indicator variables) are used for each trait, and no other information is available about error variances, it is not possible to test reciprocal causation models; and unidirectional causation models can be tested only if there are at least three sources of variation in the two traits (e.g., within-family environment, additive gene action plus dominance or within-family environment, shared environment, and additive gene action). The latter restrictions no longer apply if we are able to fit multiple indicator direction-of-causation models, and these actually fit the observed data. However, unless there are at least three sources of variation, it will not be possible to test the hypothesis of reciprocal causation.

Our analyses of statistical power confirm that samples sizes required to reject a false unidirectional causal hypothesis with a given probability are lowest (and therefore statistical power is greatest) when two correlated traits have very different modes of inheritance. Since the power of resolving additive and nonadditive genetic effects for multifactorial traits is rather low, at least as far as concerns human studies (Heath, 1983), in practice these methods will be most useful in cases where family resemblance for one trait is determined largely by genetic factors (additive genetic or, preferably, additive plus nonadditive genetic effects), and the second trait shows a strong influence of shared family environment. These will include cases where we are attempting to test causal hypotheses regarding the association between family background variables and a partly heritable disorder (cf. Parker, 1990; Plomin and Bergeman, 1991) or between such behaviors as smoking or alcohol use [which exhibit influences of both genes and shared environment (Heath, 1992; Heath et al., 1992)] and heritable personality traits, heritable differences in drug reactivity, or other variables which exhibit a strong genetic influence: Thus, in principle, many of the conditions where questions about direction of causation are difficult to address using conventional methods are precisely those where methods using cross-sectional family data will be most powerful.

Application of these methods is likely to be most trustworthy when multiple indicator models are used. Reciprocal causation models can be tested only if multiple indicators are used or if measurement errors are absent or known to be equal for both traits. We have emphasized that if a single variable is used to assess each construct, then inferences about direction of causation will be senstive to the assumption that measurement errors are uncorrelated between relatives. While it is possible to design experimental protocols to minimize this problem, including such necessary precautions as testing family members independently, it is not always possible to ensure that this ideal is achieved in all cases, particularly in large-scale (e.g., mailed questionnaire) survey research. When multiple indicators are used, it will no longer be a problem if measurement errors for a given observed variable are modestly correlated across relative pairs; but measurement error correlations between relatives across indicator variables will still cause problems. Consideration of statistical power also indicates that in the presence of measurement error, the use of multiple indicator models is important.

As with all behavioral genetic analyses, estimation of the genetic and environmental parameters of unidirectional and reciprocal causation models in any particular application rests upon strong assumptions: the assumptions of equal environmental correlations in studies of monozygotic and dizygotic twin pairs reared together or of random placement and representative sampling of genotypes and family environments in separated twin and adoption studies. Thus the validation of research findings obtained using one experimental design in a second study using a very different research design is critical. This is no less the case when we are trying to make inferences about direction of causation. Although most discussion of applications of direction of causation models to family data has occurred in the context of studies of twin pairs reared together (Heath et al., 1989a; Neale et al., 1989; Duffy and Martin, 1993), these methods can and should be applied in a variety of other experimental designs. Indeed, since separated twin designs, and adoption designs (e.g., Heath et al., 1985), are generally much more powerful means of resolving genetic 
and shared environmental influences on behavioral variation, it is likely that they will provide more powerful tests of causaul hypotheses.

\section{ACKNOWLEDGMENTS}

This research was supported by ADAMHA Grants AA03539, AA07535, AA07728, DA05588, $\mathrm{MH} 31302$, and $\mathrm{MH} 40828$. We are grateful to Michael Hodge for assistance with programming and simulation studies.

\section{REFERENCES}

Bell, R. Q. (1968). A reinterpretation of the direction of effects in studies of socialization. Psychol. Rev. 75:81-95.

Bollen, K. A. (1989). Structural Equations with Latent Variables; John Wiley and Sons, New York.

Breslau, N. (1991). Nicotine dependence, major depression, and anxiety in young adults. Arch. Gen. Psychiat. 48:1069. 1074.

Brown, G. W., and Harris, T. O. (1978). Social Origins of Depression: A Study of Psychiatric Disorder in Women, Tavistock, London.

Browne, M. W. (1984). Asymptotically distribution-free methods for the analysis of covariance structures. Br. J. Math. Stat. Psychol. 37:1-21.

Bulmer, M. G. (1980). The Mathematical Theory of Quantitative Genetics, Clarendon Press, Oxford.

Campbell, D. T. (1963). From description to experimentation: Interpreting trends from quasi-experiments. In Harris, C. W. (ed.), Problems in Measuring Change, University of Wisconsin Press, Madison, pp. 212-242.

Cardon, L. R., Fulker, D. W., and Joreskog, K. G. (1991). A LISREL model with constrained parameters for twin and adoptive families. Behav. Genet. 21:327-350.

Carey, G. (1986). Sibling imitation and contrast effects. Behav. Genet. 16:319-342.

Cavalli-Sforza, L. L., and Feldman, M. W. (1981). Cultural Transmission and Evolution: $A$ Quantitative Approach; Princeton University Press, Princeton, NJ.

Cloninger, C. R., Rice, J., and Reich, T. (1979). Multifactorial inheritance with cultural transmission and assortative mating. Am. J. Hum. Genet. 31:178-188.

Costner, H. L., and Schoenberg, R. (1973). Diagnosing indicator ills in multiple indicator models. In Goldberger, A. S., and Duncan, O. D. (eds.), Structural Equation Models in the Social Sciences, Seminar Press, New York.

Duffy, D. A., and Martin, N. G. (1993). Inferring the direction of causation in cross-sectional twin data: Theoretical and empirical considerations. Genet. Epidemiol. (in press).

Duncan, O. D. (1969). Some linear models for two-wave, twovariable panel analysis, Psychol. Bull. 72:177-182.

Eaves, L. J. (1969). The genetic analysis or continuous variation: A comparison of experimental designs applicable to human data. Br. J. Math. Stat. Psychol. 23:131-147.

Eaves, L. J. (1970). Aspects of Human Psychogenetics. Ph.D. thesis, University of Birmingham, Birmingham.

Eaves, L. J. (1976). A model for siblings effects in man. Heredity 36:205-214.

Eaves, L. J. (1977). Inferring the causes of human variation. J. Roy. Stat. Soc. A. 140:324-355.
Eaves, L. J., and Gale, J. S. (1974). A method for analyzing the genetic basis of covariation. Behav. Genet 4:253-267.

Eaves, L. J., and Eysenck, H. J. (1975), The nature of extraversion: A genetical analysis. J. Personal. Soc. Psychol. 32:102-112.

Eaves, L. J., Eysenck, H., and Martin, N. G. (1989). Genes, Culture and Personality: An Empirical Approach, Academic Press, London.

Eaves, L. J., Last, K. A., Martin, N. G., and Jinks, J. L. (1977). A progressive approach to non-additivity and genotype-environmental covariance in the analysis of human differences. Br. J. Math. Stat. Psychol. 30:1-42.

Eaves, L. J., Last, K. A., Young, P. A., and Martin, N. G. (1978). Model-fitting approaches to the analysis of human behavior. Heredity 41:249-320.

Eaves, L. J., Heath, A. C., Neale, M. C., Hewitt, J. K, and Martin, N. G. (1992). Sex differences and non-additivity in the effects of genes on personality (unpublished manuscript).

Eisen, S. A., Goldberg, J., True, W., and Henderson, W. G. (1991). A cotwin-control study of the effects of the Vietnam war on the self-reported health of veterans. $A m . J$. Epidemiol. 134:49-59.

Falconer, D. S. (1960). Quantitative Genetics, Oliver \& Boyd, Edinburgh.

Fisher, R. A. (1918). The correlation between relatives on the supposition of Mendelian inheritance. Trans. Roy. Soc. Edinburgh 53:399-433.

Fisher, R. A. (1958) Cancer and smoking. Nature 182:596.

Fulker, D. W. (1982). Extensions of the classical twin method. Proceedings of the 1981 International Congress of Human Genetics, Jerusalem, Alan R. Liss, New York.

Fulker, D. W. (1988). Genetic and cultural transmission in human behavior. In Wier, B. S., Eisen, E. J., Goodman, M. M., and Namkoong, G. (eds.), Proceedings of the Second International Conference on Quantitative $\mathrm{Ge}$ netics, Sinauer Associates, Sunderland, MA.

Fulker, D. W., and DeFries, J. C. (1983). Genetic and environmental transmission in the Colorado Adoption Project: Path analysis. Br. J. Math. Stat. Psychol. 36:175-188.

Harman, H. H. (1976). Modern Factor Analysis, University of Chicago Press, Chicago.

Heath, A. C. (1983). Human Quantitative Genetics: Some Issues and Applications, Unpublished D.Phil. dissertation, University of Oxford, Oxford.

Heath, A. C. (1990). How twin data can resolve the direction of causation: PBI and depression in American twins. Presented at Vulnerability Factors in Neurosis: What Causes What Conference, University of New South Wales, Sept.

Heath, A. C. (1993). Genetic influences on drinking behavior in humans. In Begleiter, H., and Kissin B. (eds. ), Alcohol and Alcoholism, Vol. 1, Genetic Factors and Alcoholism, Oxford University Press, Oxford (in press).

Heath, A. C., and Eaves, L. J. (1985). Resolving the effects of phenotype and social background on mate selection. Behav. Genet. 15:15-30.

Heath, A. C., and Martin, N. G. (1991a). The inheritance of alcohol sensitivity and of patterns of alcohol use. Alcohol Alcohol. Suppl. 1:141-145.

Heath, A. C., and Martin, N. G. (1991b). Intoxication after an acute dose of alcohol. An assessment of its association with alcohol consumption patterns by using twin data. Alcohol. Clin. Exp. Res. 15:122-128.

Heath, A. C., Kendler, K. S., Eaves, L. J., and Markell, D. (1985). The resolution of cultural and biological inheritance: Informativeness of different relationships. Behav. Genet. 15:439-465.

Heath, A. C., Neale, M. C., Hewitt, J. K., Eaves, L. J., and 
Fulker, D. W. (1989a). Testing structural equation models for twin data using LISREL. Behav. Genet. 19:9--35.

Heath, A. C., Eaves, L. J., and Martin, N. G. (1989b). The genetic structure of personality III. Multivariate genetic item analysis of the EPQ scales. Personal. Indiv. Diff. 10:877-888.

Heath, A. C., Cates, R., Martin, N. G., Meyer, J., Hewitt, J. K., Neale, M. C., and Eaves, L. J. (1992). Genetic contribution to risk of smoking initiation: Comparisons across birth cohorts and across cultures (submitted for publication).

Jinks, J. L., and Fulker, D. W. (1970). Comparison of the biometrical, genetical, MAVA, and classical approaches to the analysis of human behavior. Psychol. Bull. 73:311-349.

Johnston, J. J. (1972). Econometric Methods, 2nd ed., McGrawHill, New York.

Joreskog, K. (1978). The structural analysis of covariance matrices. Psychometrika 43:443-477.

Joreskog, K., and Sorbom, D. (1988). LISREL VII: Analysis of Linear Structural Relationships, Scientific Software, Mooresvill, IN.

Kendler, K. S., Heath, A. C., Martin, N. G., and Eaves, L. J. (1987). Symptoms of anxiety and symptoms of depression: Same genes, different environments? Arch. Gen. Psychiat. 44:451 457 .

Kendler, K. S., Silberg, J. L., Neale, M. C., Kessler, R. C., Heath, A. C., and Eaves, L. J. (1991). The family history method: whose psychiatric history is measured? Am. $J$. Psychiat. 148:1501-1504.

Kendler, K. S., Eaves, L. J., Neale, M. C., Heath, A. C., and Kessler, R. C. (1992). Smoking and depression: A causal analysis (submitted for publication).

Kenny, D. A. (1975). Cross-lagged panel correlation: A test for spuriousness. Psychol. Bull. 82:887-903.

Kessler, R. C. (1983). Methodological issues in the study of psychosocial stress. In Kaplan, H. B. (ed.), Psychosocial Stress: Trends in Theory and Research, Academic Press, New York.

Kessler, R. C., and Greenberg, D. F. (1981). Linear Panel Analysis: Quantitative Models for Change, Academic Press, New York.

Kessler, R. C., Kendler, K. S., Heath, A. C., Neale, M. C., and Eaves, L. J. (1992). Social support, depressed mood, and adjustment to stress: A genetic epidemiologic investigation. J. Personal. Soc. Psychol. 62:257-272.

Lange, K. L., Westlake, J., and Spence, M. A. (1976). Extension to pedigree analysis. III. Variance components by the scoring method. Ann. Hum. Genet. 39:485-491.

Locasio, J. J. (1982). The cross-lagged correlation technique: Reconsideration in terms of an exploratory utility, assumption specification and robustness. Educ. Psychol. Measure. 42:1023-1036.

Loehlin, J. C. (1978). Heredity-environment analyses of I.Q. correlations. Behav. Genet. 26:415-436.

McArdle, J. J., and Goldsmith, H. H. (1990). Alternative common-factor models for multivariate biometric analyses. Behav. Genet. 12:467-472.

Martin, N. G., and Eaves, L. J. (1977). The genetical analysis of covariance structurc. Heredity 38:79-95.

Martin, N. G., and Heath, A. C. (1991). Parental bonding and depression: What causes what? Presented at the Annual Meeting of the Behavior Genetics Association, St. Louis, MO.

Martin, N. G., Eaves, L. J., Kearsey, M. J., and Davies, P.
(1978). The power of the classical twin study. Heredity 40:97-116.

Martin, N. G., Oakeshott, J. G., Gibson, J. B., Starmer, G. A., Perl, J., and Wilks, A. V. (1985a). A twin study of psychomotor and physiological response to an acute dose of alcohol. Behav. Genet. 15:305-347.

Martin, N. G., Perl, J., Oakeshott, J. G., Gibson, J. B., Starmer, G. A., and Wilks, A. V. (1985b). A twin study of ethanol metabolism. Behav. Genet. 15:93-100.

Mather, K., and Jinks, J. L (1971). Biometrical Genetics: The Study of Continuous Variation, Chapman and Hall, London.

Matsueda, R. L., and Bielby, W. T. (1986). Statistical power in covariance structure models. In Tuma, N. B., (ed.), Sociological Methodology 1986, American Sociological Association, Washington, DC, pp. 120-158.

Neale, M. C., and Cardon, L. (1992). Methodology for Genetic Studies of Twins and Families, NATO ASI Series, Kluwer.

Neale, M. C., Heath, A. C., Hewitt, J. K., Eaves, L. J., and Fulker, D. W. (1989a). Fitting genetic models with LISREL: Hypothesis testing. Behav. Genet. 19:37-49.

Neale, M., Hewitt, J. K., Heath, A. C., and Eaves, L. J. $(1989 \mathrm{~b})$. The power of multivariate and categorical twin studies. Presented at the 6th International Congress of Twin Studies, Rome.

Neale, M. C., Walters, E., Heath, A. C., Kessler, R. C., Perusse, D., Eaves, L. J., and Kendler, K. S. (1993). Depression and parental bonding: Cause, consequence, or genetic covariance? Genet. Epidemiol. (in press).

Parker, G. (1983). Parental Overprotection: A Risk Factor in Psychosocial Development, Grune \& Stratton, New York.

Parker, G. (1990). The Parental Bonding Instrument: Psychometric properties reviewed. Psychiat. Dev. 4:317-335.

Pearson, E. S., and Hartley, E. O. (1972). Biometrika Tables for Statisticians, Cambrige University Press, Cambridge.

Plomin, R., and Bergeman, C. S. (1991). The nature of nurture: Genetic influence on environmental measures. $B e-$ hav. Brain Sci. 84:309-332.

Plomin, R., DeFries, J. C., and Loehlin, J. L. (1977). Genotype-environment interaction and correlation in the analysis of human variation. Psychol. Bull. 84:309-322.

Rice, J., Cloninger, C. R., and Reich, T. (1978). Multifactorial inheritance with cultural transmission and assortative mating. I. Description and basic properties of the unitary models. Am. J. Hum. Genet. 30:618-643.

Rogosa, D. A. (1980). A critique of cross-lagged correlation. Psychol. Bull. 88:245-258.

Satorra, A., and Saris, W. E. (1985). Power of the likelihoodratio test in covariance structure analysis. Psychometrika 50:83-90.

Schieken, R. M., Eaves, L. J., Hewitt, J. K., Mosteller, M., Bodurtha, J. N., Moscowitz, W. B., and Nance, W. E. (1989). Univariate genetic analysis of blood pressure in children (The Medical College of Virginia Twin Study). Am. J. Cardiol. 64:1333-1337.

Wright, S. (1968). Evolution and the Genetics of Populations, University of Chicago Press, Chicago.

Yamaguchi, K., and Kandel, D. B. (1984). Patterns of drug use from adolescence to adulthood. II. Sequences of progression. Am. J. Public Health 74:668-672.

Yarrow, L. J. (1963). Research in dimensions of early maternal care. Merrill-Palmer Q. 9:101-114.

Edited by N. G. Martin 